High-Order Quadratures for the Solution of Scattering Problems in Two Dimensions

Ran Duan and Vladimir Rokhlin

Technical Report YALEU/DCS/TR-1401

April 22, 2008 
We construct an iterative algorithm for the solution of forward scattering problems in two dimensions. The scheme is based on the combination of high-order quadrature formulae, fast application of integral operators in Lippmann-Schwinger equations, and the stabilized biconjugate gradient method (BI-CGSTAB). While the FFT-based fast application of integral operators and the BI-CGSTAB for the solution of linear systems are fairly standard, a large part of this paper is devoted to constructing a class of high-order quadrature formulae applicable to a wide range of singular functions in two and three dimensions; these are used to obtain rapidly convergent discretizations of Lippmann-Schwinger equations. The performance of the algorithm is illustrated with several numerical examples.

\title{
High-Order Quadratures for the Solution of Scattering Problems in Two Dimensions
}

\author{
Ran Duan and Vladimir Rokhlin \\ Technical Report YALEU/DCS/TR-1401 \\ April 22, 2008
}

The authors were supported in part by the Schlumberger Technology Corporation, ONR Grant N00014-07-1-0711, and AFOSR Grant FA9550-06-1-0239. Approved for public release: Distribution is unlimited.

Keywords: Scattering, Helmholtz, Lippmann-Schwinger, High-Order, Quadratures, Singular, Hankel 


\section{Introduction}

Forward scattering has been an active field of research in science, mathematics, and engineering over the past several decades (see e.g. [3], [4]). The most straightforward method for the solution of a forward scattering problem is to discretize the underlying PDEs directly, replace the derivatives with finite differences, and solve numerically the resulting system of linear algebraic equations. However, discretization of differential equations leads to matrices with high condition numbers, with the attendant loss of accuracy, deterioration in the performance of iterative methods, etc. Another approach is to convert the underlying PDEs into integral equations of the second kind (normally referred to as the Lippmann-Schwinger equation), discretize the latter via appropriate quadrature formulae, and deal numerically with the resulting linear systems. This paper constructs a class of high-order quadrature formulae applicable to the Lippmann-Schwinger equation in two and three dimensions.

\subsection{Statement of the Problem}

The forward scattering problem is the problem of determining the scattered field given the parameters of the scattering structure and the incident field. In this section, we formulate the two-dimensional forward scattering problem for the Helmholtz equation, and derive the corresponding Lippmann-Schwinger equation.

The forward scattering problem we investigate arises from the time domain wave equation

$$
\frac{\partial^{2}}{\partial^{2} t} \psi(x, t)=c^{2}(x) \cdot \nabla^{2} \psi(x, t)
$$

where $\psi(x, t)$ is the value of the scalar field at a point $x$ at time $t$, and $c(x)$ is the local speed of wave propagation at a point $x$. In order to solve (1), we assume that

$$
\psi(x, t)=\psi_{k}(x) e^{i k c_{0} t}
$$

where $k$ is a complex number with non-negative imaginary part, and $c_{0}$ is the speed of wave propagation outside of the scattering structure. Substituting (2) into (1), we obtain

$$
\left(\nabla^{2}+k^{2}\right) \psi_{k}(x)=k^{2} V(x) \psi_{k}(x)
$$

where

$$
V(x)=1-\left(\frac{c_{0}}{c(x)}\right)^{2} .
$$

Equation (3) is the well-known Helmholtz equation, and the operator $\left(\nabla^{2}+k^{2}\right)$ is known as the Helmholtz operator. For any point $x$ outside the scattering object, $c(x)=c_{0}$; therefore, $V(x)=0$ outside the scattering object. We represent the field $\psi_{k}(x)$ at a point $x$ as a sum of two parts: the incident field $\psi_{k}^{i n}(x)$ and the scattered field $\psi_{k}^{\text {scat }}(x)$, i.e.,

$$
\psi_{k}(x)=\psi_{k}^{i n}(x)+\psi_{k}^{s c a t}(x) .
$$

The incident field satisfies the homogenous Helmholtz equation

$$
\left(\nabla^{2}+k^{2}\right) \psi_{k}^{i n}(x)=0
$$


in some open region in $\mathbb{R}^{2}$ containing the scatterer; the scattered field satisfies the Sommerfeld radiation condition

$$
\lim _{|x| \rightarrow \infty} \sqrt{|x|}\left(\frac{\partial \psi_{k}^{\text {scat }}(x)}{\partial|x|}-i k \psi_{k}^{\text {scat }}(x)\right)=0 .
$$

Combining equations (3), (5), and (6), we obtain the equation for the scattered field

$$
\left(\nabla^{2}+k^{2}\right) \psi_{k}^{\text {scat }}(x)-k^{2} V(x) \psi_{k}^{\text {scat }}(x)=k^{2} V(x) \psi_{k}^{\text {in }}(x) .
$$

In this paper, we view the equation (8) with $\psi_{k}^{\text {scat }}$ satisfying the Sommerfeld condition (7) as the principal formulation of the forward scattering problem. A standard approach to the numerical solution of (8) is to convert (8) into the well-known Lippmann-Schwinger equation, which is an integral equation of the second kind, as follows (see, for example, [5]).

Convolving (8) with a Green's function for the equation

$$
\left(\nabla^{2}+k^{2}\right) G_{k}(x, y)=\delta(x-y)
$$

we obtain

$$
\psi_{k}^{s c a t}(x)-k^{2} \int_{D} G_{k}(x, y) V(y) \psi_{k}^{s c a t}(y) d y=k^{2} \int_{D} G_{k}(x, y) V(y) \psi_{k}^{i n}(y) d y
$$

which is an integral equation of the second kind; in (10) above, $D$ denotes the region in space where the scatterer is located. As is well-known, in two dimensions, the Green's function $G_{k}(x, y)$ satisfying the condition $(7)$ is

$$
G_{k}(x, y)=-\frac{i}{4} H_{0}^{(1)}(k\|x-y\|)
$$

where $H_{0}^{(1)}(k\|x-y\|)$ is the Hankel function of the first kind of order zero. We will define the operator $L: L^{2}(D) \rightarrow L^{2}(D)$ by the formula

$$
L(\psi)(x)=\int_{D} G_{k}(x, y) V(y) \psi(y) d y
$$

and observe that a large part of this paper is devoted to the construction of accurate discretizations of $L$.

\subsection{Overview}

A number of algorithms exist for the modeling of acoustic scattering; since we are interested in frequency domain results, we have concentrated on frequency domain (as opposed to timedomain) models. The usual approach to such problems is to convert the scattering problem into the Lippmann-Schwinger equation, and solve the latter iteratively (integral equations of the second kind being much more amenable to iterative techniques than the straightforward discretizations of underlying partial differential equations (PDEs)). In addition, the use of the Lippmann-Schwinger equation obviates the need to impose the radiation (Sommerfeld) condition at the boundary of the grid, since the "background" Green's function (11) imposes the Sommerfeld condition automatically. 
Historically, there have been two problems associated with the numerical use of integral equations in scattering calculations. First, the kernels of Lippmann-Schwinger equations are dense, except when the background is extremely attenuating; since iterative techniques require application of the matrix of the discretized integral operator to a sequence of recursively generated vectors, the cost of the procedure is prohibitive, except for extremely small-scale problems. This difficulty was overcome almost 40 years ago via the observation that the free-space Green's function for the Helmholtz equation is translation invariant; appropriately chosen discretizations of Lippmann-Schwinger equations result in Toeplitz matrices, and the latter can be rapidly applied to arbitrary vectors via the FFT (Fast Fourier Transform), resulting in algorithms with CPU time requirements proportional to $N \cdot \log (N)$, with $N$ the number of nodes in the discretization of the problem. Various forms of this approach have been widely used in electrical engineering and other fields, under the name "k-space" methods; some of the existing codes are quite fast, even for discretizations involving hundreds of millions of nodes. However, the resulting solvers for the underlying PDEs are usually not very accurate, due to the problem discussed in the following paragraph.

The second difficulty associated with numerical use of Lippmann-Schwinger equations is due to the singular character of the Green's function for the Helmholtz equation; in two dimensions, the principal term of the singularity is of the form $\log (r)$, and in three dimensions, it is of the form $1 / r$. As a result, kernels of Lippmann-Schwinger equations are singular; the singularities are located on the diagonal, and in two dimensions are of the form

$$
K(x, y)=\log (|x-y|)+P(x, y) \cdot \log (|x-y|)+Q(x, y)
$$

with $P, Q$ two smooth functions, and $P(x, x)=0$ for all $x \in R^{2}$; the corresponding form in three dimensions is

$$
K(x, y)=\frac{1}{|x-y|}+P(x, y) \cdot \frac{1}{|x-y|}+Q(x, y)
$$

It is important to observe that in most cases, we do not have access to each of the functions $P, Q$ separately, but can only evaluate the whole kernel $K$ given a pair of points $(x, y)$. In other words, standard integration techniques (such as product integration, etc.) can not be used efficiently. The standard procedure in the literature (referred to as the "singularity extraction") is to subtract the principal singularity and treat it analytically, and apply the trapezoidal quadrature rule to the remaining function. Since the latter is not smooth (having infinite derivatives at $x=y$ ), the procedure converges slowly, normally behaving like a second order scheme.

We introduce a class of quadrature formulae for functions of the form (13) in two dimensions and (14) in three dimensions. The approach is somewhat related to the Ewald summation [6], and leads to quadratures that can be viewed as a version of the corrected trapezoidal rule; it is easily combined with the FFT to obtain fast algorithms. While in principle corrections of arbitrarily high order could be constructed, in practice both the complexity of derivation and the number of corrections grow rapidly with the order. We have designed corrections of orders 4, 6, 8, and 10; they require 1, 5, 13, and 25 corrected nodes respectively.

The paper is organized as follows. In Section 2, we summarize several well-known mathematical facts to be used in the paper. In Section 3, we introduce analytical tools to be used 
in the construction of the algorithm. Section 4 describes the algorithm in detail, and contains a complexity analysis. In Section 5, several numerical examples are used to illustrate the performance of the algorithm. Finally, Section 6 contains generalizations and conclusions.

\section{Analytical Preliminaries}

In this section, we summarize several well-known mathematical facts to be used in the sections below. All of these are either well known or easily derived from well-known results.

\subsection{Notation}

For an integer $N \geq 1$, the two-dimensional discrete Fourier transform $\mathcal{F}^{N}$ is a mapping converting a two-dimensional complex sequence $a=\left\{a_{j_{1} j_{2}}\right\}, j_{1}, j_{2}=-N, \ldots, N$, into another two-dimensional complex sequence $A=\left\{A_{k_{1} k_{2}}\right\}, k_{1}, k_{2}=-N, \ldots, N$, defined by the formula

$$
A_{k_{1} k_{2}}=\sum_{j_{1}=-N}^{N} \sum_{j_{2}=-N}^{N} a_{j_{1} j_{2}} e^{-\frac{2 \pi i}{(2 N+1)} k_{1} j_{1}} e^{-\frac{2 \pi i}{(2 N+1)} k_{2} j_{2}}
$$

It is easily verified that the inverse $\left(\mathcal{F}^{N}\right)^{-1}$ of the mapping $\mathcal{F}^{N}$ is given by the formula

$$
\left(\mathcal{F}^{N}\right)^{-1}(A)_{j_{1} j_{2}}=a_{j_{1} j_{2}}=\frac{1}{(2 N+1)^{2}} \sum_{k_{1}=-N}^{N} \sum_{k_{2}=-N}^{N} A_{k_{1} k_{2}} e^{\frac{2 \pi i}{(2 N+1)} k_{1} j_{1}} e^{\frac{2 \pi i}{(2 N+1)} k_{2} j_{2}}
$$

with $j_{1}=-N, \ldots, N, j_{2}=-N, \ldots, N$.

For a Helmholtz equation

$$
\nabla^{2} \phi+k^{2} \phi=0
$$

in two dimensions, the potential $\phi$ at a point $x$ produced by a unit point source at $x_{0}$ is given by the formula

$$
\phi(x)=-\frac{i}{4} H_{0}\left(k\left\|x-x_{0}\right\|\right),
$$

where $k$ is a complex number such that $\operatorname{Im}(k) \geq 0$, and $H_{0}$ is the Hankel function of the first kind of order zero. The well-known Sommerfeld formula states that

$$
H_{0}(k r)=\frac{1}{\pi} \cdot \int_{-\infty}^{\infty} \frac{1}{\sqrt{k^{2}-\lambda^{2}}} \cdot e^{i \sqrt{k^{2}-\lambda^{2}} x} \cdot e^{i \lambda y} d \lambda
$$

for any $k \in \mathbb{C}^{+}, r, x, y \geq 0$, and $r=\sqrt{x^{2}+y^{2}}$ (see, for example, [9]).

Finally, we will need the identity

$$
\sum_{j=0}^{n} f_{j} g_{j}=f_{n} \sum_{k=0}^{n} g_{k}-\sum_{j=0}^{n-1}\left(f_{j+1}-f_{j}\right) \cdot\left(\sum_{k=0}^{j} g_{k}\right)
$$

valid for two arbitrary finite sequences $\left\{f_{j}\right\}, j=0,1,2, \ldots, n,\left\{g_{j}\right\}, j=0,1,2, \ldots, n$, . By analogy with integration by parts, (20) is normally referred to as summation by parts; it is easily verified by a substitution. 


\subsection{High-Order Corrected Trapezoidal Quadrature Rules for Sin- gular Functions in One Dimension}

For a function $f:[a, b] \rightarrow R^{1}$ and integer $n \geq 2$, the $n$-point trapezoidal rule $T_{n}$ is defined by the formula

$$
T_{n}(f)=h\left(\sum_{i=0}^{n-1} f(a+i h)-\left(\frac{f(a)+f(b)}{2}\right)\right)
$$

where

$$
h=\frac{b-a}{n-1}
$$

and is widely used as an approximation to the integral $\int_{a}^{b} f(x) d x$. It is second order convergent, as long as the second derivative of $f$ is continuous on $[a, b]$. In other words, if $f \in C^{2}[a, b]$, then

$$
\int_{a}^{b} f(x) d x=T_{n}(f)+O\left(h^{2}\right) .
$$

For any function $f \in C^{2 m+2}[a, b]$ with integer $m \geq 1$, the well-known Euler-Maclaurin formula (see, for example, [1]) states that there exists a real number $\xi$ with $a<\xi<b$, such that

$$
\int_{a}^{b} f(x) d x=T_{n}(f)+\sum_{l=1}^{m} \frac{h^{2 l} B_{2 l}}{(2 l) !}\left(f^{(2 l-1)}(b)-f^{(2 l-1)}(a)\right)-\frac{h^{2 m+2} B_{2 m+2}}{(2 m+2) !} f^{(2 m+2)}(\xi),
$$

where $B_{k}, k=0,1,2, \ldots$ denote the Bernoulli numbers (see [1]). It is easily seen from (24) that for any function $f \in C^{m}[a-m h, b+m h]$ with integer $m \geq 3$, it is possible to construct quadratures of the form

$$
T_{\beta^{m}}^{n}(f)=T_{n}(f)+h \sum_{k=-\frac{m-1}{2}}^{\frac{m-1}{2}}(f(b+k h)-f(a+k h)) \beta_{k}^{m},
$$

where $\beta_{k}^{m}$ are coefficients such that

$$
\int_{a}^{b} f(x) d x=T_{\beta^{m}}^{n}(f)+O\left(h^{m+1}\right)
$$

with $h$ defined by $(22)$. The quadrature $T_{\beta^{m}}^{n}$ is referred to as the $(m+1)^{t h}$-order endpointcorrected trapezoidal rule; for any given $k$ and $m$, where $m \geq 3$, and is odd, the coefficient $\beta_{k}^{m}$ can be obtained via a direct calculation (see [7]).

While (21) and (25) are widely used for the numerical integration of smooth functions, their use for singular integrands tends to encounter difficulties (see, for example, [11]). In [7], a class of quadrature formulae is constructed for approximating integrals of singular functions of the form

$$
f(x)=\phi(x) s(x)
$$


where $\phi \in C^{m}[-b-m h, b+m h]$ with $m \geq 3$, and $s$ is an integrable function on $[-b, b]$ with a singularity at zero. For integers $n \geq 1, p \geq 1$, and odd $m \geq 3$, the quadrature $U_{\tau^{h} \beta^{m}}$ for the functions with separable singularities (i.e., functions defined by (27)) is given by the formula

$$
U_{\tau^{h} \beta^{m}}(f)=T_{\beta^{m}}^{\prime n}(f)+\sum_{j=-p}^{p} \tau_{j}^{h} \phi(j h) .
$$

$\operatorname{In}(28)$,

$$
T_{\beta^{m}}^{\prime n}(f)=h\left(\sum_{\substack{j=-n \\ j \neq 0}}^{n} f(j h)-\left(\frac{f(-b)+f(b)}{2}\right)\right)+h \sum_{k=-\frac{m-1}{2}}^{\frac{m-1}{2}}(f(b+k h)-f(-b+k h)) \beta_{k}^{m},
$$

where the coefficients $\beta_{k}^{m}$ can be obtained via direct calculation (see [7]), $h=\frac{b}{n}$, and the coefficients $\tau_{j}^{h}$ can be obtained by solving the system of linear equations

$$
\sum_{j=-p}^{p} x_{j}^{i-1} \tau_{j}^{h}=\int_{-b}^{b}\left(x^{i-1} s(x)\right) d x-T_{\beta^{m}}^{\prime n}\left(x^{i-1} s\right),
$$

with $x_{j}=j h$, and $i=1,2, \ldots, 2 p+1$. The quadrature formula (28) is of order $2 p+2$.

Remark 2.1 While (21) is the standard trapezoidal rule, and (25) is the trapezoidal rule with endpoints corrections, (28) is the trapezoidal rule with corrections at both endpoints and a singular point inside the interval. Here, the singular point is the center point, so the scheme is sometimes called a "center-corrected trapezoidal rule." The coefficients $\tau_{j}^{h}$ are called correction coefficients.

Remark 2.2 The only difference between $T_{\beta^{m}}^{\prime n}(f)$ and $T_{\beta^{m}}^{n}(f)$ is that $T_{\beta^{m}}^{\prime n}(f)$ does not contain the term $h \cdot f(0)$, which may become infinite for $f$ of the form (27).

\subsection{Toeplitz Convolution}

This section introduces two-dimensional Toeplitz convolutions and a procedure for the calculation of two-dimensional Toeplitz convolutions via the two-dimensional discrete Fourier transform. The Toeplitz convolution $a * b$ of finite two-dimensional complex sequences $a=\left\{a_{j_{1} j_{2}}\right\}, j_{1}, j_{2}=-N, \ldots, N$, and $b=\left\{b_{j_{1} j_{2}}\right\}, j_{1}, j_{2}=-2 N, \ldots, 2 N$, is defined by the formula

$$
(a * b)_{k_{1} k_{2}}=\sum_{j_{1}=-N}^{N} \sum_{j_{2}=-N}^{N} a_{j_{1} j_{2}} b_{k_{1}-j_{1}, k_{2}-j_{2}},
$$

where $k_{1}, k_{2}=-N, \ldots, N$. The well-known convolution theorem states that the Toeplitz convolution $a * b$ is equal to the inverse Fourier transform of the product of the Fourier transform of $a^{\prime}$ and $b$, where $a^{\prime}$ is a two-dimensional sequence obtained by padding the two-dimensional sequence $a$ with zeros. In other words,

$$
(a * b)_{k_{1} k_{2}}=\left(\mathcal{F}^{2 N}\right)^{-1}\left(\mathcal{F}^{2 N}\left(a^{\prime}\right) \cdot \mathcal{F}^{2 N}(b)\right)_{k_{1} k_{2}},
$$


where $k_{1}, k_{2}=-N, \ldots, N$, and the coefficients of the two-dimensional complex sequence $a^{\prime}=\left\{a_{i_{1} i_{2}}^{\prime}\right\}, i_{1}, i_{2}=-2 N, \ldots, 2 N$ are defined by the formulae

$$
a_{i_{1} i_{2}}^{\prime}=a_{i_{1} i_{2}}
$$

when $-N \leq i_{1}, i_{2} \leq N$, otherwise

$$
a_{i_{1} i_{2}}^{\prime}=0
$$

Remark 2.3 While direct calculation of Toeplitz convolution (31) leads to time cost of order $O\left(N^{4}\right)$, which is prohibitive for large scale problems, application of FFT to the formula (32) reduces the time cost to $O\left(N^{2} \cdot \log N\right)$ (see, for example, [2]). In this paper, FFT is used for the fast calculation of Toeplitz convolution.

\section{Mathematical Apparatus}

In this section, we introduce analytical tools to be used in the construction of the algorithms.

\subsection{Endpoint Corrected Trapezoidal Quadrature Rules in Two Di- mensions}

This section can be viewed as the extension of results of Section 2.2 to two dimensions.

For a function $f:[a, b] \times[a, b] \rightarrow R^{1}$ and integer $n \geq 2$, the two-dimensional trapezoidal rule $T_{n}^{2 D}$ is defined by the formula

$$
T_{n}^{2 D}(f)=\sum_{i=0}^{n-1} \sum_{j=0}^{n-1} f(a+i h, a+j h) \cdot h^{2} \cdot \beta_{i j},
$$

where

$$
h=\frac{b-a}{n-1}
$$

and $\beta_{i j}$ equals 1 in the interior of the square $[a, b] \times[a, b]$, equals $\frac{1}{2}$ in the interior of the edge, and equals $\frac{1}{4}$ on the corners of the square.

Further, if $f \in C^{2}[a, b] \times[a, b]$, then

$$
\int_{a}^{b} \int_{a}^{b} f(x, y) d x d y=T_{n}^{2 D}(f)+O\left(h^{2}\right) .
$$

The proof consists of a straightforward application of one-dimensional trapezoidal rule (21) to both directions in two dimensions, and thus omitted.

The following Lemma provides the two-dimensional version of the Euler-Maclaurin formula. 
Lemma 3.1 Suppose that a function $f \in C^{2 m+2}[a, b] \times[a, b]$, integers $m \geq 1, n \geq 2$. Then,

$$
\begin{aligned}
& \int_{a}^{b} \int_{a}^{b} f(x, y) d x d y=T_{n}^{2 D}(f)+\sum_{i=0}^{i=n-1} \sum_{l=1}^{m} \frac{h^{2 l+1} \cdot B_{2 l} \cdot \beta_{i}}{(2 l) !} \cdot \\
& \left(\frac{\partial^{2 l-1}}{\partial x^{2 l-1}} f(b, a+i h)-\frac{\partial^{2 l-1}}{\partial x^{2 l-1}} f(a, a+i h)-\frac{\partial^{2 l-1}}{\partial y^{2 l-1}} f(a+i h, b)-\frac{\partial^{2 l-1}}{\partial y^{2 l-1}} f(a+i h, a)\right) \\
& +\sum_{l=1}^{m} \sum_{l^{\prime}=1}^{m} \frac{h^{2 l+2 l^{\prime}} \cdot B_{2 l} \cdot B_{2 l^{\prime}}}{(2 l !)\left(2 l^{\prime} !\right)} \cdot\left(\frac{\partial^{2 l+2 l^{\prime}-2}}{\partial x^{2 l-1} \partial y^{2 l^{\prime}-1}}(f(b, b)+f(a, a)-f(a, b)-f(b, a))\right)+O\left(h^{2 m+2}\right),
\end{aligned}
$$

where $T_{n}^{2 D}(f)$ is defined in (35), $h$ is defined in (36),

$$
\beta_{i}=\left\{\begin{array}{ll}
1 & 0<i<n-1 \\
1 / 2 & i=0 \text { or } i=n-1
\end{array},\right.
$$

and $B_{k}, k=0,1,2, \ldots$ denote the Bernoulli numbers.

Proof. The Euler-Maclaurin formula (24) can be rewritten as

$$
\int_{a}^{b} f(x) d x=T_{n}(f)+\sum_{l=1}^{m} \frac{h^{2 l} B_{2 l}}{(2 l) !}\left(f^{(2 l-1)}(b)-f^{(2 l-1)}(a)\right)+O\left(h^{2 m+2}\right) .
$$

Hence,

$$
\begin{aligned}
& \int_{a}^{b} \int_{a}^{b} f(x, y) d x d y= \\
& \quad \int_{a}^{b}\left(\sum_{i=0}^{n-1} f(a+i h, y) \cdot \beta_{i}+\sum_{l=1}^{m} \frac{h^{2 l} B_{2 l}}{(2 l) !}\left(f^{(2 l-1)}(b)-f^{(2 l-1)}(a)\right)+O\left(h^{2 m+2}\right)\right) d y .
\end{aligned}
$$

The conclusion of the Lemma follows immediately from applying the formula (40) to the integrals in (41).

The following Lemma provides a $(2 m+2)^{t h}$-order endpoint-corrected trapezoidal rule in two dimensions.

Lemma 3.2 Suppose that a function $f \in C^{2 m+2}[a, b] \times[a, b]$ with integers $m \geq 1, n \geq 2$, $h=\frac{b-a}{n-1}$. Then,

$$
\int_{a}^{b} \int_{a}^{b} f(x, y) d x d y=T_{\beta^{2 m+1}}^{2 D, n}(f)+O\left(h^{2 m+2}\right)
$$

In (42),

$$
\begin{aligned}
& T_{\beta^{2 m+1}}^{2 D, n}(f)=T_{n}^{2 D}(f)+h^{2} \sum_{i=0}^{n-1} \sum_{k=-m}^{m}\left(\beta_{k}^{2 m+1} \beta_{i} .\right. \\
& (f(b+k h, i h)+f(i h, b+k h)-f(a+k h, i h)-f(i h, a+k h)))+h^{2} \sum_{k=-m}^{m} \sum_{k^{\prime}=-m}^{m}\left(\beta_{k}^{2 m+1} \beta_{k^{\prime}}^{2 m+1}\right. \\
& \left.\left(f\left(a+k h, a+k^{\prime} h\right)+f\left(b+k h, b+k^{\prime} h\right)-f\left(a+k h, b+k^{\prime} h\right)-f\left(b+k h, a+k^{\prime} h\right)\right)\right),
\end{aligned}
$$


$T_{n}^{2 D}, \beta_{k}^{2 m+1}$, and $\beta_{i}$ are defined by (35), (25) and (39).

Proof. It is easily observed from the formulae (24), (25) that

$$
\sum_{l=1}^{m} \frac{h^{2 l+1} B_{2 l}}{(2 l !)} f^{(2 l-1)}(a)=\sum_{k=-m}^{m} f(a+k h) \cdot \beta_{k}^{2 m+1} \cdot h^{2} .
$$

Therefore,

$$
\sum_{k=-m}^{m} h^{2} \cdot f(a+k h, a+i h) \cdot \beta_{k}^{2 m+1}=\sum_{l=1}^{m} \frac{h^{2 l+1} B_{2 l}}{(2 l !)} \frac{\partial^{2 l+1}}{\partial x^{2 l+1}} f(a, a+i h),
$$

and

$$
\begin{array}{r}
\sum_{l=1}^{m} \sum_{l^{\prime}=1}^{m} \frac{h^{2 l+2 l^{\prime}} \cdot B_{2 l} \cdot B_{2 l^{\prime}}}{(2 l !)\left(2 l^{\prime} !\right)}\left(\frac{\partial^{2 l+2 l^{\prime}-2}}{\partial x^{2 l-1} \partial y^{2 l^{\prime}-1}} f(a, a)\right) \\
=\sum_{l^{\prime}=1}^{m} \frac{h^{2 l^{\prime}} B_{2 l^{\prime}}}{\left(2 l^{\prime} !\right)} \cdot\left(\sum_{k=-m}^{m} h \cdot \beta_{k}^{2 m+1} \frac{\partial^{2 l^{\prime}-1}}{\partial y^{2 l^{\prime}-1}} f(a+k h, a)\right) \\
=\sum_{k=-m}^{m} h^{2} \beta_{k}^{2 m+1} \cdot\left(\sum_{l^{\prime}=1}^{m} \frac{h^{2 l^{\prime}-1} B_{2 l^{\prime}}}{\left(2 l^{\prime} !\right)} \cdot \frac{\partial^{2 l^{\prime}-1}}{\partial y^{2 l^{\prime}-1}} f(a+k h, a)\right) \\
=\sum_{k=-m}^{m} \sum_{k^{\prime}=-m}^{m} h^{2} \cdot \beta_{k}^{2 m+1} \beta_{k^{\prime}}^{2 m+1} f\left(a+k h, a+k^{\prime} h\right) .
\end{array}
$$

Now, (43) follows immediately from the combination of (45), (46).

\subsection{High-Order Center Corrected Trapezoidal Quadrature Rules for the Singular Functions in Two Dimensions}

The following Lemma provides an estimate of the difference between the integral and the end-point corrected trapezoidal quadrature for functions of the form $x^{2 p+2} \cdot \log \left(x^{2}+y^{2}\right)$.

Lemma 3.3 Suppose that $n$ is a positive integer, $a, h$ are two positive real numbers such that $h=a / n$, and integers $m, p$ are such that $m \geq p+1 \geq 1$. Then,

$$
\int_{-a}^{a} \int_{-a}^{a}\left(x^{2 p+2} \cdot \log \left(x^{2}+y^{2}\right)\right) d x d y=T_{\beta^{2 m+1}}^{2 D, n}\left(x^{2 p+2} \cdot \log \left(x^{2}+y^{2}\right)\right)+O\left(h^{2 p+4}\right),
$$

with $T_{\beta^{2 m+1}}^{2 D, n}$ defined by (43).

Proof. Here, we prove the case of $p=0$ as an illustration; the proof for $p>0$ is quite similar. For simplicity, we will be denoting $x^{2} \cdot \log \left(x^{2}+y^{2}\right)$ by $g(x, y)$. Then,

$$
\frac{\partial g(x, y)}{\partial y}=\frac{2 x^{2} y}{x^{2}+y^{2}}
$$




$$
\frac{\partial^{3} g(x, y)}{\partial y^{3}}=\frac{16 x^{2} y^{3}}{\left(x^{2}+y^{2}\right)^{3}}-\frac{12 x^{2} y}{\left(x^{2}+y^{2}\right)^{2}}
$$

and

$$
\frac{\partial^{6} g(x, y)}{\partial y^{6}}=-\frac{7680 x^{2} y^{6}}{\left(x^{2}+y^{2}\right)^{6}}+\frac{11520 x^{2} y^{4}}{\left(x^{2}+y^{2}\right)^{5}}-\frac{4320 x^{2} y^{2}}{\left(x^{2}+y^{2}\right)^{4}}+\frac{240 x^{2}}{\left(x^{2}+y^{2}\right)^{3}} .
$$

Replacing the derivatives in (24) with the appropriate finite differences, we rewrite (24) in the form

$$
\begin{aligned}
& \int_{a}^{b} g(x) d x=T_{n}(g)+\frac{1}{12} h \cdot(-g(b+h)+g(a+h)+g(b-h)-g(a-h))+ \\
& C_{1} \cdot h^{4} \cdot\left(g^{(3)}(b)-g^{(3)}(a)\right)-C_{2} \cdot h^{6} \cdot g^{(6)}(\xi) \\
&=T_{\beta^{3}}^{n}(g)+C_{1} \cdot h^{4} \cdot\left(g^{(3)}(b)-g^{(3)}(a)\right)+C_{2} \cdot h^{6} \cdot g^{(6)}(\xi)
\end{aligned}
$$

where $C_{1}$ and $C_{2}$ are two constants independent of $g$, and $\xi \in[a, b]$. Therefore, for any fixed $x$ and $g(x, y)=x^{2} \cdot \log \left(x^{2}+y^{2}\right)$, the error $\epsilon(x)$ of the end-point corrected trapezoidal quadrature in the $y$ direction is

$$
\epsilon(x)=\int_{-a}^{a} g(x, y) d y-T_{\beta^{3}}^{n}(g)=C_{1} \cdot h^{4}\left(\frac{16 x^{2} a^{3}}{\left(x^{2}+a^{2}\right)^{3}}-\frac{12 x^{2} a}{\left(x^{2}+a^{2}\right)^{2}}\right)+C_{2} \cdot h^{6} \cdot g^{(6)}(x, \xi) .
$$

Summing up all the errors along the $x$ axis, we obtain

$$
\begin{array}{r}
\epsilon=\int_{-a}^{a} \int_{-a}^{a}\left(x^{2} \cdot \log \left(x^{2}+y^{2}\right)\right) d x d y-T_{\beta^{3}}^{2 D, n}\left(x^{2} \cdot \log \left(x^{2}+y^{2}\right)\right) \sim 2 \cdot \sum_{i=1}^{n} h \cdot \epsilon(i h) \\
\leq h \cdot \sum_{i=1}^{n}\left(C_{1} \cdot h^{4}\left(\frac{16(i h)^{2} a^{3}}{\left((i h)^{2}+a^{2}\right)^{3}}-\frac{12(i h)^{2} a}{\left((i h)^{2}+a^{2}\right)^{2}}\right)+h \cdot \sum_{i=1}^{n} C_{2} \cdot h^{6} \cdot\left|g^{(6)}(\xi)\right|_{\text {max }}\right. \\
\leq C_{1} h^{4} \int_{0}^{a}\left(\frac{16 x^{2} a^{3}}{\left(x^{2}+a^{2}\right)^{3}}-\frac{12 x^{2} a}{\left(x^{2}+a^{2}\right)^{2}}\right) d x+C_{2} \cdot h^{7} \sum_{i=1}^{n} \frac{240}{(i h)^{4}} \\
\sim C_{1} \cdot(3-\pi) \cdot h^{4}+C_{2} \cdot h^{6} \cdot \log (h) \sim O\left(h^{4}\right) .
\end{array}
$$

Theorem 3.4 below is an extension of the formula (28) to two dimensions. For an integer $p \geq 0$, it supplies a $(2 p+4)^{t h}$-order center-corrected quadrature formula on $\mathbb{R}^{2}$ for the functions of the form

$$
f(x, y)=\phi(x, y) \cdot s(x, y)
$$

where $\phi: R^{2} \rightarrow R$, and

$$
s(x, y)=\log \left(x^{2}+y^{2}\right)+P(x, y) \cdot \log \left(x^{2}+y^{2}\right)+Q(x, y),
$$

with $P, Q$ two smooth functions, and $P(0,0)=0$. Suppose that $n, m$ are positive integers, and $a, h$ are two positive real numbers such that $h=a / n$. We define $T_{\beta^{2 m+1}}^{2 D, n}(f)$ by the 
formula

$$
\begin{aligned}
& T_{\beta^{2 m+1}}^{2 D, n}(f)=\sum_{(i, j) \in M} f(i h, j h) \cdot h^{2} \cdot \beta_{i j}+h^{2} \sum_{i=-n}^{n} \sum_{k=-m}^{m}\left(\beta_{k}^{2 m+1} \beta_{i} .\right. \\
& (f(a+k h, i h)+f(i h, a+k h)-f(-a+k h, i h)-f(i h,-a+k h)))+h^{2} \sum_{k=-m}^{m} \sum_{k^{\prime}=-m}^{m}\left(\beta_{k}^{2 m+1} \beta_{k^{\prime}}^{2 m+1} .\right. \\
& \left.\left(f\left(-a+k h,-a+k^{\prime} h\right)+f\left(a+k h, a+k^{\prime} h\right)-f\left(-a+k h, a+k^{\prime} h\right)-f\left(a+k h,-a+k^{\prime} h\right)\right)\right),
\end{aligned}
$$

where

$$
\begin{gathered}
M=\{i, j \in \mathbb{Z}:|i| \leq n,|j| \leq n,(i, j) \neq(0,0)\}, \\
\beta_{i}=\left\{\begin{array}{ll}
1 & |i|<n \\
1 / 2 & |i|=n
\end{array} ;\right.
\end{gathered}
$$

in (56), coefficients $\beta_{i j}$ equal 1 in the interior of the square $[-a, a] \times[-a, a]$, equal $\frac{1}{2}$ in the interior of the edge, and equal $\frac{1}{4}$ on the corners of the square, and $\beta_{k}^{2 m+1}$ are defined in (25).

Theorem 3.4 Suppose that $\phi \in C^{2 p+2}\left(\mathbb{R}^{2}\right)$ with $p \geq 0$, integer $m \geq p+1$, and $s$ is a singular function on $R^{2}$ with a logarithmic singularity at $(0,0)$, i.e., of the form (55). Suppose further that

$$
U_{\tau^{h} \beta^{2 m+1}}^{2 D}(\phi \cdot s)=T_{\beta^{2 m+1}}^{2 D, n}(\phi \cdot s)+\sum_{(i, j) \in W} \tau_{i j}^{h} \phi(i h, j h),
$$

where $T_{\beta^{2 m+1}}^{2 D, n}(\phi \cdot s)$ is defined by the formula (56),

$$
W=\{i, j \in \mathbb{Z}:|i+j| \leq p \text { and }|i-j| \leq p\},
$$

and the coefficients $\tau_{i j}^{h}$ in (59) satisfy the system of linear equations

$$
\sum_{(i, j) \in W} x_{i}^{i^{\prime}-1} y_{j}^{j^{\prime}-1} \tau_{i j}^{h}=\int_{-a}^{a} \int_{-a}^{a}\left(x^{i^{\prime}-1} y^{j^{\prime}-1} s(x, y)\right) d x d y-T_{\beta^{2 m+1}}^{2 D, n}\left(x^{i^{\prime}-1} y^{j^{\prime}-1} s\right),
$$

with $x_{i}=i h, y_{j}=j h$, and $\left(i^{\prime}, j^{\prime}\right) \in H$, where $H=\left\{i^{\prime}, j^{\prime}, \in Z: i^{\prime} \geq 1, j^{\prime} \geq 1, i^{\prime}+j^{\prime} \leq 2 p+2\right\}$. Then,

$$
\int_{-a}^{a} \int_{-a}^{a}(\phi(x, y) \cdot s(x, y)) d x d y=U_{\tau^{h} \beta^{2 m+1}}^{2 D}(\phi \cdot s)+O\left(h^{2 p+4}\right) .
$$

Proof. Applying the Taylor expansion to the function $\phi(x, y)$ at the point $(0,0)$ we have

$$
\phi(x, y)=P(x, y)+R(x, y)
$$

where

$$
P(x, y)=\left.\sum_{j=0}^{2 p+1} \sum_{i=0}^{j} \frac{1}{j !}\left(\begin{array}{l}
j \\
i
\end{array}\right) x^{i} y^{j-i} \frac{\partial^{j}}{\partial x^{i} \partial y^{j-i}} \phi(x, y)\right|_{x=0, y=0}
$$


and

$$
R(x, y)=\left.\frac{1}{(2 p+2) !} \sum_{i=0}^{2 p+2}\left(\begin{array}{c}
2 p+2 \\
i
\end{array}\right) x^{i} y^{2 p+2-i} \frac{\partial^{2 p+2}}{\partial x^{i} \partial y^{2 p+2-i}} \phi(x, y)\right|_{x=\xi_{1}, y=\xi_{2}},
$$

where $\xi_{1}, \xi_{2} \in[-a, a] \times[-a, a]$. Thus,

$$
\begin{aligned}
\left|\int_{-a}^{a} \int_{-a}^{a}(\phi(x, y) \cdot s(x, y)) d x d y-U_{\tau^{h} \beta^{2 m+1}}^{2 D}(\phi \cdot s)\right| \\
\qquad\left|\int_{-a}^{a} \int_{-a}^{a}(P(x, y) \cdot s(x, y)) d x d y-U_{\tau^{h} \beta^{2 m+1}}^{2 D}(P \cdot s)\right|+ \\
\quad\left|\int_{-a}^{a} \int_{-a}^{a}(R(x, y) \cdot s(x, y)) d x d y-U_{\tau^{h} \beta^{2 m+1}}^{2 D}(R \cdot s)\right| .
\end{aligned}
$$

Now, we make the following three observations. Due to (61),

$$
\int_{-a}^{a} \int_{-a}^{a}(P(x, y) \cdot s(x, y)) d x d y-U_{\tau^{h} \beta^{2 m+1}}^{2 D}(P \cdot s)=0
$$

and due to (59),

$$
\begin{aligned}
\mid \int_{-a}^{a} \int_{-a}^{a}( & R(x, y) \cdot s(x, y)) d x d y-U_{\tau^{h} \beta^{2 m+1}}^{2 D}(R \cdot s) \mid \\
& \leqslant\left|\int_{-a}^{a} \int_{-a}^{a}(R(x, y) \cdot s(x, y)) d x d y-T_{\beta^{2 m+1}}^{2 D, n}(R \cdot s)\right|+\left|\sum_{(i, j) \in W} \tau_{i j}^{h} R(i h, j h)\right| .
\end{aligned}
$$

Due to formulae (47) and (61), it is obvious that

$$
\left|\int_{-a}^{a} \int_{-a}^{a}(R(x, y) \cdot s(x, y)) d x d y-T_{\beta^{2 m+1}}^{2 D, n}(R \cdot s)\right| \sim O\left(h^{2 p+4}\right)
$$

and the coefficients $\tau_{i j}^{h}$ are of the order $h^{2}$, hence,

$$
\left|\sum_{(i, j) \in W} \tau_{i j}^{h} R(i h, j h)\right| \sim O\left(h^{2 p+4}\right) .
$$

Finally, the conclusion of the Lemma follows from the combination of $(66)-(70)$.

\subsection{High-Order Center Corrected Trapezoidal Quadrature Rules for the Green's Function for the Helmholtz equation}

In this section, we prove Theorem 3.10, which is the principal analytical tool of this paper. Theorem 3.10 describes the $10^{t h}$-order center-corrected trapezoidal quadrature formulae for

the Hankel function. It can be viewed as a special case of Theorem 3.4 with $p=3$, and the singular function $s$ the Hankel function $H_{0}$. 
In the remainder of this paper, we will be using the following notation. For any $k \in C^{+}$ and $h>0$, we will define the complex numbers $D_{0}, D_{1}, D_{2}, D_{3}, D_{4}, D_{5}$, via the formulae

$$
\begin{gathered}
D_{0}=\int_{-\infty}^{\infty} \int_{-\infty}^{\infty} H_{0}^{(1)}(k r) d x d y-\sum_{(p, q) \neq(0,0)} H_{0}^{(1)}\left(k \sqrt{(p h)^{2}+(q h)^{2}}\right) \cdot h^{2}, \\
D_{1}=\int_{-\infty}^{\infty} \int_{-\infty}^{\infty} H_{0}^{(1)}(k r) x^{2} d x d y-\sum_{(p, q) \neq(0,0)} H_{0}^{(1)}\left(k \sqrt{(p h)^{2}+(q h)^{2}}\right) \cdot(p h)^{2} \cdot h^{2}, \\
D_{2}=\int_{-\infty}^{\infty} \int_{-\infty}^{\infty} H_{0}^{(1)}(k r) x^{4} d x d y-\sum_{(p, q) \neq(0,0)} H_{0}^{(1)}\left(k \sqrt{(p h)^{2}+(q h)^{2}}\right) \cdot(p h)^{4} \cdot h^{2}, \\
D_{3}=\int_{-\infty}^{\infty} \int_{-\infty}^{\infty} H_{0}^{(1)}(k r) x^{2} y^{2} d x d y-\sum_{(p, q) \neq(0,0)} H_{0}^{(1)}\left(k \sqrt{(p h)^{2}+(q h)^{2}}\right) \cdot(p h)^{2}(q h)^{2} \cdot h^{2}, \\
D_{4}=\int_{-\infty}^{\infty} \int_{-\infty}^{\infty} H_{0}^{(1)}(k r) x^{6} d x d y-\sum_{(p, q) \neq(0,0)} H_{0}^{(1)}\left(k \sqrt{(p h)^{2}+(q h)^{2}}\right) \cdot(p h)^{6} \cdot h^{2}, \\
D_{5}=\int_{-\infty}^{\infty} \int_{-\infty}^{\infty} H_{0}^{(1)}(k r) x^{4} y^{2} d x d y-\sum_{(p, q) \neq(0,0)} H_{0}^{(1)}\left(k \sqrt{(p h)^{2}+(q h)^{2}}\right) \cdot(p h)^{4}(q h)^{2} \cdot h^{2} .
\end{gathered}
$$

The following Lemma is a simple consequence of the Sommerfeld formula (19).

Lemma 3.5 For any $k \in \mathbb{C}^{+}, r, x, y \geq 0$, and $r=\sqrt{x^{2}+y^{2}}$,

$$
H_{0}(k r)=\frac{1}{\pi} \cdot \int_{-\infty}^{\infty} \frac{1}{\sqrt{k^{2}-\lambda^{2}}} \cdot e^{i \cdot \frac{\sqrt{2}}{2} \cdot\left(\sqrt{k^{2}-\lambda^{2}}-\lambda\right) \cdot x} \cdot e^{i \cdot \frac{\sqrt{2}}{2} \cdot\left(\sqrt{k^{2}-\lambda^{2}}+\lambda\right) \cdot y} d \lambda
$$

where $r^{2}=x^{2}+y^{2}, x, y \geq 0$.

The following two technical lemmas follow immediately from the Sommerfeld formula (19).

Lemma 3.6 For any $k \in \mathbb{C}^{+}$, and $a \geq 0$,

$$
\begin{aligned}
& \int_{-a}^{a} \int_{-a}^{a} H_{0}(k r) d x d y=\frac{4}{\pi} \cdot \int_{-\infty}^{\infty} \frac{1}{\sqrt{k^{2}-\lambda^{2}}} \cdot \\
& \frac{e^{i \cdot \frac{\sqrt{2}}{2} \cdot\left(\sqrt{k^{2}-\lambda^{2}}-\lambda\right) \cdot a}-1}{i \cdot \frac{\sqrt{2}}{2} \cdot\left(\sqrt{k^{2}-\lambda^{2}}-\lambda\right)} \cdot \frac{e^{i \cdot \frac{\sqrt{2}}{2} \cdot\left(\sqrt{k^{2}-\lambda^{2}}+\lambda\right) \cdot a}-1}{i \cdot \frac{\sqrt{2}}{2} \cdot\left(\sqrt{k^{2}-\lambda^{2}}+\lambda\right)} d \lambda,
\end{aligned}
$$

with $r=\sqrt{x^{2}+y^{2}}$.

Proof. Substituting (77) into the left side of (78), and changing the order of integration, we obtain

$$
\begin{aligned}
\int_{-a}^{a} \int_{-a}^{a} H_{0} & (k r) d x d y=4 \int_{0}^{a} \int_{0}^{a} H_{0}(k r) d x d y \\
= & \frac{4}{\pi} \int_{-\infty}^{\infty} \frac{d \lambda}{\sqrt{k^{2}-\lambda^{2}}} \cdot \int_{0}^{a} e^{i \cdot \frac{\sqrt{2}}{2} \cdot\left(\sqrt{k^{2}-\lambda^{2}}-\lambda\right) \cdot x} d x \cdot \int_{0}^{a} e^{i \cdot \frac{\sqrt{2}}{2} \cdot\left(\sqrt{k^{2}-\lambda^{2}}+\lambda\right) \cdot y} d y \\
& =\frac{4}{\pi} \cdot \int_{-\infty}^{\infty} \frac{1}{\sqrt{k^{2}-\lambda^{2}}} \cdot \frac{e^{i \cdot \frac{\sqrt{2}}{2} \cdot\left(\sqrt{k^{2}-\lambda^{2}}-\lambda\right) \cdot a}-1}{i \cdot \frac{\sqrt{2}}{2} \cdot\left(\sqrt{k^{2}-\lambda^{2}}-\lambda\right)} \cdot \frac{e^{i \cdot \frac{\sqrt{2}}{2} \cdot\left(\sqrt{k^{2}-\lambda^{2}}+\lambda\right) \cdot a}-1}{i \cdot \frac{\sqrt{2}}{2} \cdot\left(\sqrt{k^{2}-\lambda^{2}}+\lambda\right)} d \lambda
\end{aligned}
$$


Lemma 3.7 For any $k \in \mathbb{C}^{+}$, integer $n \geq 1$, and $a>0$,

$$
\begin{aligned}
& \sum_{p=-n}^{n} \sum_{q=-n}^{n} H_{0}\left(k \sqrt{(p h)^{2}+(q h)^{2}}\right) \cdot h^{2} \cdot \beta_{p q} \\
& =\frac{4}{\pi} \int_{-\infty}^{\infty} \frac{1}{\sqrt{k^{2}-\lambda^{2}}} \cdot h^{2} \cdot\left(\frac{e^{i \cdot \frac{\sqrt{2}}{2} \cdot\left(\sqrt{k^{2}-\lambda^{2}}-\lambda\right) \cdot a}-1}{e^{i \cdot \frac{\sqrt{2}}{2} \cdot\left(\sqrt{k^{2}-\lambda^{2}}-\lambda\right) \cdot h}-1}-\frac{1}{2}+\frac{1}{2} e^{i \cdot \frac{\sqrt{2}}{2} \cdot\left(\sqrt{k^{2}-\lambda^{2}}-\lambda\right) \cdot a}\right) \\
& \cdot\left(\frac{e^{i \cdot \frac{\sqrt{2}}{2} \cdot\left(\sqrt{k^{2}-\lambda^{2}}+\lambda\right) \cdot a}-1}{e^{i \cdot \frac{\sqrt{2}}{2} \cdot\left(\sqrt{k^{2}-\lambda^{2}}+\lambda\right) \cdot h}-1}-\frac{1}{2}+\frac{1}{2} e^{i \cdot \frac{\sqrt{2}}{2} \cdot\left(\sqrt{k^{2}-\lambda^{2}}+\lambda\right) \cdot a}\right) d \lambda,
\end{aligned}
$$

with

$$
h=a / n,
$$

and $\beta_{p q}$ equals 1 in the interior of the $(2 n+1) \times(2 n+1)$ square, equals $\frac{1}{2}$ in the interior of the edge, and equals $\frac{1}{4}$ on the corners of the square.

Proof. The trapezoidal sum (84) over the domain $[-a, a] \times[-a, a]$ is equal to four times the trapezoidal sum over the domain $[0, a] \times[0, a]$. In other words,

$$
\begin{aligned}
& \sum_{p=-n}^{n} \sum_{q=-n}^{n} H_{0}^{(1)}\left(k \sqrt{(p h)^{2}+(q h)^{2}}\right) \cdot h^{2} \cdot \beta_{p q} \\
& =4 \cdot \sum_{p=0}^{n} \sum_{q=0}^{n} H_{0}^{(1)}\left(k \sqrt{(p h)^{2}+(q h)^{2}}\right) \cdot h^{2} \cdot \beta_{p q}^{\prime}
\end{aligned}
$$

where $\beta_{p q}^{\prime}$ equals 1 in the interior of the $(n+1) \times(n+1)$ square, equals $\frac{1}{2}$ in the interior of the edge, and equals $\frac{1}{4}$ on the corners of the square. Substituting (77) into (83), and exchanging the order of integration and summation, we obtain

$$
\begin{aligned}
& \sum_{p=-n}^{n} \sum_{q=-n}^{n} H_{0}\left(k \sqrt{(p h)^{2}+(q h)^{2}}\right) \cdot h^{2} \cdot \beta_{p q} \\
& \frac{4}{\pi} \int_{-\infty}^{\infty} \frac{d \lambda}{\sqrt{k^{2}-\lambda^{2}}} \cdot h^{2} \cdot\left(\sum_{p=0}^{n} e^{i \cdot \frac{\sqrt{2}}{2} \cdot\left(\sqrt{k^{2}-\lambda^{2}}-\lambda\right) \cdot p \cdot h}-\frac{1+e^{i \cdot \frac{\sqrt{2}}{2} \cdot\left(\sqrt{k^{2}-\lambda^{2}}-\lambda\right) \cdot a}}{2}\right) \\
& \cdot\left(\sum_{q=0}^{n} e^{i \cdot \frac{\sqrt{2}}{2} \cdot\left(\sqrt{k^{2}-\lambda^{2}}+\lambda\right) \cdot q \cdot h}-\frac{1+e^{i \cdot \frac{\sqrt{2}}{2} \cdot\left(\sqrt{k^{2}-\lambda^{2}}+\lambda\right) \cdot a}}{2}\right) \\
& =\frac{4}{\pi} \int_{-\infty}^{\infty} \frac{1}{\sqrt{k^{2}-\lambda^{2}}} \cdot h^{2} \cdot\left(\frac{e^{i \cdot \frac{\sqrt{2}}{2} \cdot\left(\sqrt{k^{2}-\lambda^{2}}-\lambda\right) \cdot a}-1}{e^{i \cdot \frac{\sqrt{2}}{2} \cdot\left(\sqrt{k^{2}-\lambda^{2}}-\lambda\right) \cdot h}-1}-\frac{1}{2}+\frac{1}{2} e^{i \cdot \frac{\sqrt{2}}{2} \cdot\left(\sqrt{k^{2}-\lambda^{2}}-\lambda\right) \cdot a}\right) \\
& \cdot\left(\frac{e^{i \cdot \frac{\sqrt{2}}{2} \cdot\left(\sqrt{k^{2}-\lambda^{2}}+\lambda\right) \cdot a}-1}{e^{i \cdot \frac{\sqrt{2}}{2} \cdot\left(\sqrt{k^{2}-\lambda^{2}}+\lambda\right) \cdot h}-1}-\frac{1}{2}+\frac{1}{2} e^{i \cdot \frac{\sqrt{2}}{2} \cdot\left(\sqrt{k^{2}-\lambda^{2}}+\lambda\right) \cdot a}\right) d \lambda .
\end{aligned}
$$


Remark 3.1 As $a \rightarrow \infty$, the exponential terms $e^{\left(i \cdot \frac{\sqrt{2}}{2} \cdot\left(\sqrt{k^{2}-\lambda^{2}} \pm \lambda\right) \cdot a\right)}$ in $(78)$ and (81) tend to zero; this fact will be used in Lemma 3.8 below.

The following lemma provides an analytical form for the difference between the integral (78) and the trapezoidal sum (81). Its proof consists of combining Remark 3.1 with (78), (81).

Lemma 3.8 For any $k \in \mathbb{C}^{+}$and $h>0$,

$$
\begin{aligned}
\int_{-\infty}^{\infty} \int_{-\infty}^{\infty} H_{0}^{(1)}(k r) d x d y & -\sum_{p=-\infty}^{\infty} \sum_{q=-\infty}^{\infty} H_{0}^{(1)}\left(k \sqrt{(p h)^{2}+(q h)^{2}}\right) \cdot h^{2} \\
& =\frac{4}{\pi} \int_{-\infty}^{\infty} \frac{d \lambda}{\sqrt{k^{2}-\lambda^{2}}} \cdot\left(\frac{1}{\left(i \alpha_{1}\right)\left(i \alpha_{2}\right)}-\frac{h^{2}}{4} \frac{e^{i \alpha_{1} h}+1}{e^{i \alpha_{1} h}-1} \frac{e^{i \alpha_{2} h}+1}{e^{i \alpha_{2} h}-1}\right),
\end{aligned}
$$

where

$$
\begin{gathered}
r=\sqrt{x^{2}+y^{2}} \\
\alpha_{1}=\frac{\sqrt{2}}{2}\left(\sqrt{k^{2}-\lambda^{2}}-\lambda\right), \alpha_{2}=\frac{\sqrt{2}}{2}\left(\sqrt{k^{2}-\lambda^{2}}+\lambda\right) .
\end{gathered}
$$

The following Lemma follows immediately from Lemma 3.8. It supplies an analytical form for the difference

$\int_{-\infty}^{\infty} \int_{-\infty}^{\infty}\left(x^{i-1} y^{j-1} H_{0}\left(k \sqrt{x^{2}+y^{2}}\right)\right) d x d y-\sum_{(p, q) \neq(0,0)}\left((p h)^{i-1}(q h)^{j-1} H_{0}\left(k \sqrt{(p h)^{2}+(q h)^{2}}\right)\right) \cdot h^{2}$,

with $i=1, j=1$.

Remark 3.2 (90) is the right hand side of the equation (61), and thus is directly used in the calculation of coefficients $\tau_{i j}^{h}$. Direct numerical subtraction of the integral and the sum in (90) leads to loss of accuracy because of cancellation errors, especially when $i, j$ are relatively large. Lemma 3.9 below and Lemmas $6.1-6.5$ in Appendix A, provide analytical formulae for $(90)$ with $(i, j)=\{(1,1),(3,1),(5,1),(3,3),(7,1),(5,3)\}$, i.e., $D_{0}-D_{5}$ defined by $(71)-$ (76), so that cancellation errors are reduced.

Lemma 3.9 For any $k \in \mathbb{C}^{+}$and $h>0$,

$$
\begin{aligned}
& D_{0}=\int_{-\infty}^{\infty} \int_{-\infty}^{\infty} H_{0}^{(1)}(k r) d x d y-\sum_{(p, q) \neq(0,0)} H_{0}^{(1)}\left(k \sqrt{(p h)^{2}+(q h)^{2}}\right) \cdot h^{2} \\
& =\frac{4}{\pi} \int_{-\infty}^{\infty} \frac{d \lambda}{\sqrt{k^{2}-\lambda^{2}}} \cdot\left(\frac{1}{\left(i \alpha_{1}\right)\left(i \alpha_{2}\right)}-\frac{h^{2}}{2} \cdot \frac{e^{i \alpha_{1} h}+e^{i \alpha_{2} h}}{\left(e^{i \alpha_{1} h}-1\right)\left(e^{i \alpha_{2} h}-1\right)}\right) \\
& =\frac{4}{\pi} \int_{-\infty}^{\infty} \frac{d \lambda}{\sqrt{k^{2}-\lambda^{2}}} \cdot \frac{\left(\frac{\lambda^{2}}{4}-\frac{k^{2}}{24}\right) h^{2}+z_{1}+z_{2}+i \sqrt{\frac{1}{2}} \lambda h\left(y_{1}-y_{2}\right)+y_{1} y_{2}}{\left(i \alpha_{1}\right)\left(i \alpha_{2}\right)\left(1+x_{1}\right)\left(1+x_{2}\right)}
\end{aligned}
$$

where the complex numbers $x_{1}, x_{2}, y_{1}, y_{2}, z_{1}, z_{2}$ are defined by the formulae

$$
x_{1}=\frac{e^{i \alpha_{1} h}-1}{i \alpha_{1} h}-1=\sum_{n=1}^{\infty} \frac{\left(i \alpha_{1} h\right)^{n}}{(n+1) !}, \quad x_{2}=\frac{e^{i \alpha_{2} h}-1}{i \alpha_{2} h}-1=\sum_{n=1}^{\infty} \frac{\left(i \alpha_{2} h\right)^{n}}{(n+1) !},
$$




$$
\begin{array}{cc}
y_{1}=x_{1}-\frac{i \alpha_{1} h}{2}=\sum_{n=2}^{\infty} \frac{\left(i \alpha_{1} h\right)^{n}}{(n+1) !}, & y_{2}=x_{2}-\frac{i \alpha_{2} h}{2}=\sum_{n=2}^{\infty} \frac{\left(i \alpha_{2} h\right)^{n}}{(n+1) !} \\
z_{1}=y_{1}-\frac{\left(i \alpha_{1} h\right)^{2}}{6}=\sum_{n=3}^{\infty} \frac{\left(i \alpha_{1} h\right)^{n}}{(n+1) !}, & z_{2}=y_{2}-\frac{\left(i \alpha_{2} h\right)^{2}}{6}=\sum_{n=3}^{\infty} \frac{\left(i \alpha_{2} h\right)^{n}}{(n+1) !}
\end{array}
$$

and $r, \alpha_{1}$, and $\alpha_{2}$ are defined by (88), (89).

Proof. Substituting (77) into

$$
\sum_{(p, q) \neq(0,0)} H_{0}^{(1)}\left(k \sqrt{(p h)^{2}+(q h)^{2}}\right) \cdot h^{2},
$$

and exchanging the order of integration and summation, we obtain

$$
\begin{aligned}
& \sum_{(p, q) \neq(0,0)} H_{0}^{(1)}\left(k \sqrt{(p h)^{2}+(q h)^{2}}\right) \cdot h^{2} \\
= & \frac{4}{\pi} \int_{-\infty}^{\infty} \frac{d \lambda}{\sqrt{k^{2}-\lambda^{2}}} \cdot\left(\frac{h^{2}}{4} \frac{e^{i \alpha_{1} h}+1}{e^{i \alpha_{1} h}-1} \frac{e^{i \alpha_{2} h}+1}{e^{i \alpha_{2} h}-1}-\frac{h^{2}}{4}\right) \\
= & \frac{4}{\pi} \int_{-\infty}^{\infty} \frac{d \lambda}{\sqrt{k^{2}-\lambda^{2}}} \cdot \frac{h^{2}}{2} \cdot \frac{e^{i \alpha_{1} h}+e^{i \alpha_{2} h}}{\left(e^{i \alpha_{1} h}-1\right)\left(e^{i \alpha_{2} h}-1\right)} .
\end{aligned}
$$

Now, (91) follows immediately from the combination of (97), (78), and Remark 3.1. Substituting (93) into (91), we obtain

$$
\begin{aligned}
& \frac{1}{\left(i \alpha_{1}\right)\left(i \alpha_{2}\right)}-\frac{h^{2}}{2} \cdot \frac{e^{i \alpha_{1} h}+e^{i \alpha_{2} h}}{\left(e^{i \alpha_{1} h}-1\right)\left(e^{i \alpha_{2} h}-1\right)} \\
& =\frac{1}{\left(i \alpha_{1}\right)\left(i \alpha_{2}\right)}-\frac{1}{2} \cdot \frac{2+i \alpha_{1} h\left(1+x_{1}\right)+i \alpha_{2} h\left(1+x_{2}\right)}{\left(i \alpha_{1}\right)\left(i \alpha_{2}\right)\left(1+x_{1}\right)\left(1+x_{2}\right)} \\
& =\frac{x_{1}+x_{2}+x_{1} x_{2}-\frac{1}{2} i \alpha_{1} h\left(1+x_{1}\right)-\frac{1}{2} i \alpha_{1} h\left(1+x_{1}\right)}{\left(i \alpha_{1}\right)\left(i \alpha_{2}\right)\left(1+x_{1}\right)\left(1+x_{2}\right)} .
\end{aligned}
$$

Finally, (92) follows from the combination of (93), (94), (95) and (98).

Remark 3.3 Introducing the notation $z=\frac{\lambda}{k}$, we rewrite $D_{0}$ in the form

$$
\begin{aligned}
& D_{0}=\int_{-\infty}^{\infty} \int_{-\infty}^{\infty} H_{0}^{(1)}(k r) d x d y-\sum_{(p, q) \neq(0,0)} H_{0}^{(1)}\left(k \sqrt{(p h)^{2}+(q h)^{2}}\right) \cdot h^{2} \\
= & \frac{4 h^{2}}{\pi} \int_{-\infty}^{\infty} \frac{d z}{\sqrt{1-z^{2}}} \cdot\left(\frac{1}{\left(z^{2}-0.5\right) \cdot(k h)^{2}}-\frac{1}{2} \cdot \frac{e^{i \frac{\sqrt{2}}{2}\left(\sqrt{1-z^{2}}-z\right) k h}+e^{i \frac{\sqrt{2}}{2}\left(\sqrt{1-z^{2}}+z\right) k h}}{\left(e^{i \frac{\sqrt{2}}{2}\left(\sqrt{1-z^{2}}-z\right) k h}-1\right)\left(e^{i \frac{\sqrt{2}}{2}\left(\sqrt{1-z^{2}}+z\right) k h}-1\right)}\right) .
\end{aligned}
$$

Thus, $D_{0}$ is entirely determined by $k$ and $h$, and is of the form $h^{2} \cdot f(k \cdot h)$. Similarly, $D_{1}$ is of the form $h^{4} \cdot f(k \cdot h) ; D_{2}$ and $D_{3}$ are of the form $h^{6} \cdot f(k \cdot h) ; D_{4}$ and $D_{5}$ are of the form $h^{8} \cdot f(k \cdot h)$. In other words, except for the multiplicative factors $\left(h^{2}, h^{4}, h^{6}\right.$, or $\left.h^{8}\right), D_{0}-D_{5}$ only depend on the product $k \cdot h$. 
Remark 3.4 Even when Lemmas 6.1 - 6.5 are used, a certain loss of accuracy in the calculation of $D_{1}-D_{5}$ is encountered (see Remark 3.2 above). Thus, evaluating $D_{0}$ in double precision, one obtains roughly 13 digits; for $D_{1}$ one gets 9 digits, and $D_{2} D_{3} D_{4} D_{5}$ yield even fewer digits.

To avoid this difficulty, we utilized extended (real *32) precision to precompute the coefficients $D_{0}-D_{5}$ for values of $k h$ at appropriately chosen nodes on the boundary of the square $\Omega=[0,1] \times[0,1]$ in the complex plane, and used Lagrange interpolation to evaluate $D_{0}$ - $D_{5}$ for arbitrary points in $\Omega$ to 13 digits (see [8] for a detailed description of the technique). Thus, in all of our numerical experiments reported in Section 5 below, the coefficients $D_{0}-$ $D_{5}$ were obtained by interpolation, rather than computed "from scratch".

Now, we are ready to formulate Theorem 3.10, which is the principal analytical tool of this paper (together with Lemmas 6.6, 6.7, 6.8). Theorem 3.10 describes the $10^{\text {th }}$-order center-corrected quadrature formula for the Green's function for the Helmholtz equation in two dimensions; this theorem is a special case of the high-order center-corrected trapezoidal rule for singular functions in two dimensions (see Theorem 3.4) with $p=3$, and $s(x, y)=$ $H_{0}^{(1)}\left(k \sqrt{x^{2}+y^{2}}\right)$. The $4^{\text {th }}$-order, $6^{\text {th }}$-order, and $8^{\text {th }}$-order center-corrected quadratures are similar and listed in Appendix B (see Lemma 6.6, 6.7 and 6.8). All the proofs are quite similar to that of Theorem 3.4, and are omitted.

Theorem 3.10 Suppose that $n \geq 1$ is an integer, and $a, h$ are two positive real numbers such that $h=a / n$. Suppose further that $\phi: \mathbb{R}^{2} \rightarrow \mathbb{C}$ is a function such that $\phi \in c^{8}(R \times R)$, and that $\phi$ is zero outside the square $[-a, a] \times[-a, a]$. Then, for any $k \in \mathbb{C}^{+}$,

$$
\int_{-a}^{a} \int_{-a}^{a} \phi(x, y) \cdot H_{0}\left(k \sqrt{x^{2}+y^{2}}\right) d x d y=U_{\tau^{h}}\left(\phi \cdot H_{0}\right)+O\left(h^{10}\right) .
$$

In (100),

$$
U_{\tau^{h}}\left(\phi \cdot H_{0}\right)=T^{2 D}\left(\phi \cdot H_{0}\right)+\sum_{p, q \in S} \tau_{p q}^{h} \phi(p h, q h),
$$

where

$$
\begin{aligned}
S & =\{p, q \in Z:|p+q| \leq 3 \text { and }|p-q| \leq 3\} \\
T^{\prime 2 D}\left(\phi \cdot H_{0}\right) & =\sum_{(p, q) \neq(0,0)}\left(\phi(p h, q h) \cdot H_{0}^{(1)}\left(k \sqrt{(p h)^{2}+(q h)^{2}}\right)\right) \cdot h^{2}
\end{aligned}
$$

and

$$
\begin{gathered}
\tau_{00}^{h}=D_{0}-\frac{49}{18} \frac{D_{1}}{h^{2}}+\frac{7}{9} \frac{D_{2}}{h^{4}}+\frac{3}{2} \frac{D_{3}}{h^{4}}-\frac{1}{18} \frac{D_{4}}{h^{6}}-\frac{1}{2} \frac{D_{5}}{h^{6}} \\
\tau_{ \pm 10}^{h}=\tau_{0 \pm 1}^{h}=\frac{3}{4} \frac{D_{1}}{h^{2}}-\frac{13}{48} \frac{D_{2}}{h^{4}}-\frac{19}{24} \frac{D_{3}}{h^{4}}+\frac{1}{48} \frac{D_{4}}{h^{6}}+\frac{7}{24} \frac{D_{5}}{h^{6}} \\
\tau_{ \pm 20}^{h}=\tau_{0 \pm 2}^{h}=-\frac{3}{40} \frac{D_{1}}{h^{2}}+\frac{1}{12} \frac{D_{2}}{h^{4}}+\frac{1}{24} \frac{D_{3}}{h^{4}}-\frac{1}{120} \frac{D_{4}}{h^{6}}-\frac{1}{24} \frac{D_{5}}{h^{6}} \\
\tau_{ \pm 30}^{h}=\tau_{0 \pm 3}^{h}=\frac{1}{180} \frac{D_{1}}{h^{2}}-\frac{1}{144} \frac{D_{2}}{h^{4}}+\frac{1}{720} \frac{D_{4}}{h^{6}} \\
\tau_{ \pm 1 \pm 1}^{h}=\frac{5}{12} \frac{D_{3}}{h^{4}}-\frac{1}{6} \frac{D_{5}}{h^{6}}
\end{gathered}
$$




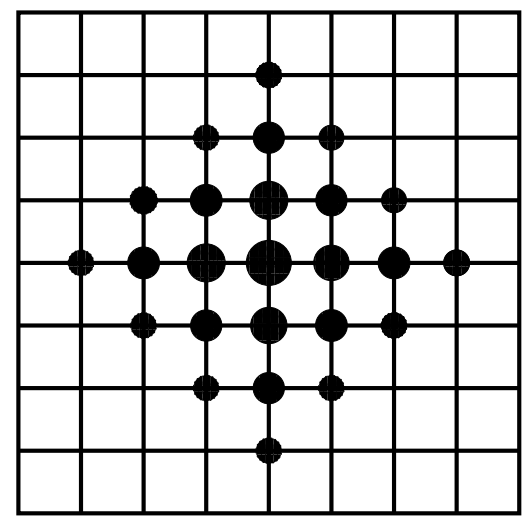

Figure 1: The 25 correction nodes

$$
\tau_{ \pm 1 \pm 2}^{h}=\tau_{ \pm 2 \pm 1}^{h}=-\frac{1}{48} \frac{D_{3}}{h^{4}}+\frac{1}{48} \frac{D_{5}}{h^{6}} .
$$

Remark 3.5 For simplicity, we assume here the function $\phi$ to be zero outside the square $[-a, a] \times[-a, a]$. Thus, the endpoint corrected trapezoidal rule $T^{\prime 2 D, n}{ }^{2 m+1}\left(\phi \cdot H_{0}\right)$ in Theorem 3.4 reduces to the standard trapezoidal rule $T^{2 D}\left(\phi \cdot H_{0}\right)$, and the integral and the sum on the square $[-a, a] \times[-a, a]$ are identical to those in $R^{2}$. This simplification allows the direct use of the analytical formulae for $D_{0}-D_{5}$ (see Lemma 3.9 above and Lemmas $6.1-6.5$ in Appendix A).

Remark 3.6 Combining Remark 3.3 with the definitions (104)-(109), we observe that each of the coefficients $\tau_{p q}^{h}$ in (104)-(109) has the form $h^{2} \cdot f(k \cdot h)$; we will refer to the coefficients $\tau_{p q}^{h}$ as correction coefficients.

Remark 3.7 The set $S$ defined in (102) contains 25 pairs of integers $(p, q)$; in other words, corrections at 25 points around the singularity are required to construct a $10^{\text {th }}$-order quadrature formula (see Figure 1). In general, for any integer $p \geq 0,2 p^{2}+2 p+1$ correction nodes are needed to obtain a quadrature of order $2 p+4$.

\subsection{Fast Numerical Application of Discretized Lippmann-Schwinger Operators}

In this section, we combine the $10^{\text {th }}$-order quadrature formula for the integral (100) with the FFT to obtain a fast procedure for the application of discretizations of the operator (12). We will denote by $D$ the square $[-a, a] \times[-a, a]$ in $R^{2}$.

Suppose that $N \geq 1$ is an integer, $h$ is a positive real number, and $S$ is a set defined in (102). Suppose further that the coefficients $\tau_{i_{1} i_{2}}^{h}$ are defined in (104)-(109). Then, we define a two-dimensional complex sequence $H=\left\{H_{i_{1} i_{2}}\right\}, i_{1}, i_{2}=-2 N, \ldots, 2 N$, as follows:

$$
H_{i_{1} i_{2}}=H_{0}\left(k \sqrt{\left(i_{1} h\right)^{2}+\left(i_{2} h\right)^{2}}\right)+\tau_{i_{1} i_{2}}^{h} / h^{2},
$$


when $\left(i_{1}, i_{2}\right) \in S$ and $\left(i_{1}, i_{2}\right) \neq(0,0)$;

$$
H_{00}=\tau_{00}^{h} / h^{2}
$$

and

$$
H_{i_{1} i_{2}}=H_{0}\left(k \sqrt{\left(i_{1} h\right)^{2}+\left(i_{2} h\right)^{2}}\right),
$$

otherwise. We define a complex sequence $\Phi=\left\{\Phi_{i_{1} i_{2}}\right\}, i_{1}, i_{2}=-N, \ldots, N$, to be the twodimensional sequence defined by the formula

$$
\Phi_{i_{1} i_{2}}=\phi\left(i_{1} h, i_{2} h\right)
$$

where $\phi: \mathbb{R}^{2} \rightarrow \mathbb{C}$ is a two-dimensional $c^{8}$-function which is zero outside $D$.

Lemma 3.11 Suppose that the integers $n, l_{1}, l_{2}$ are such that $n \geq 1,-n \leq l_{1} \leq n,-n \leq l_{2} \leq$ $n$, and that the real numbers $a, x, y$ are such that $a>0,-a \leq x \leq a,-a \leq y \leq a$. Suppose further that $\phi: \mathbb{R}^{2} \rightarrow \mathbb{C}$ is a $c^{8}$-function which is zero outside the square $[-a, a] \times[-a, a]$. Then for any $k \in \mathbb{C}^{+}$,

$$
\begin{aligned}
& \int_{-a}^{a} \int_{-a}^{a} \phi\left(x^{\prime}, y^{\prime}\right) \cdot H_{0}^{(1)}\left(k \sqrt{\left(x-x^{\prime}\right)^{2}+\left(y-y^{\prime}\right)^{2}}\right) d x^{\prime} d y^{\prime} \\
& =\sum_{-n \leq i_{1} \leq n} \sum_{-n \leq i_{2} \leq n} \Phi_{i_{1} i_{2}} \cdot H_{\left(l_{1}-i_{1}\right)\left(l_{2}-i_{2}\right)}+O\left(h^{10}\right),
\end{aligned}
$$

where $h=a / n, x=l_{1} h, y=l_{2} h$, the two-dimensional sequence $\Phi=\left\{\Phi_{i_{1} i_{2}}\right\}, i_{1}, i_{2}=-n, \ldots, n$ is defined in (113), and the two-dimensional sequence $H=\left\{H_{j_{1} j_{2}}\right\}, j_{1}, j_{2}=-2 n, \ldots, 2 n$ is defined in (110) - (112).

Proof. Due to (100), (101),

$$
\begin{aligned}
& \int_{-a}^{a} \phi\left(x^{\prime}, y^{\prime}\right) \cdot H_{0}^{(1)}\left(k \sqrt{\left(x-x^{\prime}\right)^{2}+\left(y-y^{\prime}\right)^{2}}\right) d x^{\prime} d y^{\prime} \\
& =\sum_{\left(i_{1}, i_{2}\right) \in I^{\prime}} \phi\left(i_{1} h, i_{2} h\right) \cdot H_{0}^{(1)}\left(k \sqrt{\left(l_{1} h-i_{1} h\right)^{2}+\left(l_{2} h-i_{2} h\right)^{2}}\right) \cdot h^{2} \\
& +\sum_{(p, q) \in S} \tau_{p q}^{h} \phi\left(l_{1} h+p h, l_{2} h+q h\right)+O\left(h^{10}\right)
\end{aligned}
$$

where

$$
I^{\prime}=\left\{i_{1}, i_{2} \in \mathbb{Z}:\left|i_{1}\right| \leq n,\left|i_{2}\right| \leq n,\left(i_{1}, i_{2}\right) \neq\left(l_{1}, l_{2}\right)\right\}
$$

and $S$ is defined in (102). Now, (114) follows immediately from the combination of (115) and the definitions in (110) - (113).

Remark 3.8 Obviously, (114) is the Toeplitz convolution of the two-dimensional sequences $\Phi, H$, and as such, it can be rapidly calculated via the FFT (see Section 2.3 above). Thus,

$$
\sum_{-n \leq i_{1} \leq n} \sum_{-n \leq i_{2} \leq n} \Phi_{i_{1} i_{2}} \cdot H_{\left(l_{1}-i_{1}\right)\left(l_{2}-i_{2}\right)}=\left(\mathcal{F}^{2 n}\right)^{-1}\left(\mathcal{F}^{2 n}\left(\Phi^{\prime}\right) \cdot \mathcal{F}^{2 n}(H)\right)_{l_{1} l_{2}},
$$


where $-n \leq l_{1} \leq n,-n \leq l_{2} \leq n$, and the two-dimensional sequence $\Phi^{\prime}=\left\{\Phi_{i j}^{\prime}\right\}, i, j=$ $-2 n, \ldots, 2 n$, is defined by

$$
\Phi_{i j}^{\prime}= \begin{cases}\Phi_{i j} & \text { if }|i| \leq n \text { and }|j| \leq n \\ 0 & \text { if }|i|>n \text { or }|j|>n\end{cases}
$$

Remark 3.9 For any point $x$ outside the square $[-a, a] \times[-a, a]$, integral (12) is approximated via the standard trapezoidal rule. This approximation is $10^{\text {th }}$-order convergent, as long as $\phi \in c^{10}\left(R^{2}\right)$.

\section{Description of the Procedure}

This section describes the algorithm of the present paper in some detail. We start with an informal description, follow with a more detailed one, and finish with a complexity analysis.

\subsection{Informal Description of the Algorithm}

Below, we describe an FFT-based $10^{\text {th }}$-order iterative algorithm for the solution of the Lippmann-Schwinger equation

$$
\psi(x)-k^{2} \int_{D} G_{k}(x, y) V(y) \psi(y) d y=k^{2} \int_{D} G_{k}(x, y) V(y) \phi(y) d y
$$

in two dimensions, where $D=[-a, a] \times[-a, a], G_{k}$ is the Green's function for the Helmholtz equation in two dimension, i.e., $G_{k}(x, y)=-\frac{i}{4} \cdot H_{0}^{(1)}(k\|x-y\|)$, and $V(x)$ denotes the potential at a point $x$. Here, $\psi(x)$ and $\phi(x)$ are the scattered and the incident fields at a point $x$, respectively.

As discussed in Remark 3.9, once the scattered field $\psi$ in the domain $D$ is known, the scattered field $\psi$ outside $D$ can be calculated via the standard trapezoidal rule applied to (119). Therefore, we focus on obtaining the solution of (119) for $x \in D$. Obviously, (119) can be written as the linear system

$$
(\mathbb{1}-A) \psi=A \phi
$$

where $\psi$ is the unknown scattered field in $D, \phi$ is the given incident field in $D, \mathbb{1}$ is the identity operator, and $A$ is the integral operator in (119). As discussed in Section 3, we use (114) to approximate the integral operator $A$ on the functions $\psi, \phi$. With the help of FFT (see Remark 3.8), we apply the discretized version of $A$ rapidly to arbitrary vectors, and solve the linear system (120) iteratively. We use one of the most popular iterative solvers, BI-CGSTAB (the stabilized bi-conjugate gradient method) (see [10], [12]).

\subsection{Detailed Description of the Algorithm}

Comment [Choose principal parameters.] 
Set the size of the scattering structure to $[-a, a] \times[-a, a]$.

Set the initial position of a point source to $\left(x_{0}, y_{0}\right)$ to generate the incident field.

Choose precision $\epsilon$ to be achieved for the iterative solver.

Choose an integer $n$; set $h=\frac{a}{n}$; set the number of nodes discretizing a side of the square to $2 n+1$, so that the total number of nodes in the discretization is $N=(2 n+1)^{2}$.

Choose the wave number $k$ for the incident and the scattered fields.

Construct a two-dimensional sequence $\left\{V_{i j}\right\}, i, j=-n, \ldots, n$ via the formula $V_{i j}=$ $V(i h, j h)$.

\section{Stage 1}

Comment [Construct the values of the the Green's function.]

For the user-specified $h$ and $k$, calculate the correction coefficients $D_{0}, D_{1}, D_{2}, D_{3}, D_{4}, D_{5}$ in (71)-(76) via interpolation (see Remark 3.4).

Construct the two-dimensional sequence $H$ via the formulae $(110)-(112)$ on the square $[-2 a, 2 a] \times[-2 a, 2 a]$, and calculate its Fourier transform using the two-dimensional FFT.

\section{Stage 2}

Comment [Construct the right hand side of the linear system (120).]

For a point source $\left(x_{0}, y_{0}\right)$, construct a two-dimensional sequence $\Phi=\left\{\Phi_{i j}\right\}, i, j=$ $-n, \ldots, n$ for the discretized incident field on the domain $[-a, a] \times[-a, a]$ via the formula (113). Construct the two-dimensional sequence $f=\left\{\Phi_{i j} \cdot V_{i j}\right\}, i, j=-n, \ldots, n$.

As in Remark 3.8, use the two-dimensional FFT to calculate the Toeplitz convolution of the sequences $H$ and $f$.

\section{Stage 3}

Comment [Solve the linear system using iterative solvers.]

Use the iterative solver BI-CGSTAB to solve the linear system $(\mathbb{1}-A) \psi=A \phi$ to the predetermined precision $\epsilon$. The multiplication $A \psi$ is done via the combination of FFT and the Toeplitz convolution of the two-dimensional sequences $H$ and $g$, where $g=\left\{\Psi_{i j} \cdot V_{i j}\right\}$, $i, j=-n, \cdots, n$ with $\Psi_{i j}=\psi(i h, j h)$ (see Remark 3.8).

The solution is the scattered field at the $N$ discretization points in the square $[-a, a] \times$ $[-a, a]$.

\section{Stage 4}

Comment [Calculate the scattered field at any point in the two-dimensional plane.]

Use interpolation to obtain the scattered field at any arbitrary point in the square $[-a, a] \times$ $[-a, a]$, based on the scattered field at the $N$ discretization points. As in Remark 3.9, apply the trapezoidal rule to (119) to obtain the scattered field at any arbitrary point outside the square $[-a, a] \times[-a, a]$. 


\subsection{Complexity Analysis}

A brief analysis of the complexity of the algorithm is given below.

In stage 1, the construction of the two-dimensional sequence $H \operatorname{costs} O(N)$, where $N$ is the total number of the discretization points on the square $[-a, a] \times[-a, a]$, i.e., $N=(2 n+1)^{2}$. The two-dimensional FFT costs $O(N \log (N))$. Thus, the CPU time cost of the stage 1 is of the order $O(N \log (N))$.

In stage 2 , the construction of the two dimensional sequences $\Phi, f$ costs $O(N)$, and the two-dimensional FFT costs $O(N \log (N))$. Thus, the CPU time cost of the stage 2 is of order $O(N \log (N))$.

The CPU time cost of the stage 3 is of order $O\left(N_{\text {iter }} \cdot N \log (N)\right)$, where $N_{\text {iter }}$ is the number of iterations required by the iterative solver to get the pre-determined precision $\epsilon$.

In stage 4 , the CPU time cost of interpolating the field at any point in $[-a, a] \times[-a, a]$ $O(N)$.

Summing up the CPU times above, we obtain the time estimate for the algorithm

$$
T=\alpha\left(N_{i t e r} \cdot N \log (N)\right)+\beta \cdot N+\gamma,
$$

where $N$ is the total number of discretization points, $N_{i t e r}$ is the number of iterations required by the iterative solvers to reach the precision $\epsilon$, and the coefficients $\alpha, \beta, \gamma$ are determined by the computer system, implementation, etc.

The storage requirements of the algorithm are determined by the total number of discretization points $N$ and the number of iterations $K$ performed before restarting the iterative solvers, and are of the form

$$
S=O(K \cdot N)
$$

\section{$5 \quad$ Numerical Examples}

The algorithm of Section 4 has been implemented in FORTRAN 77 in double precision. In this section, we illustrate the performance of the scheme as applied to two scattering objects: a Gaussian and a crude model of the human skull. The experiments were carried out on a $2.8 \mathrm{GHz}$ Pentium D desktop with $2 \mathrm{~Gb}$ of RAM and an L2 cache of $1 \mathrm{Mb}$. The calculations reported in Tables 1 and 3 were carried out with a requested accuracy of $10^{-13}$; the calculations reported in Tables 2 and 4 were carried out with a requested accuracy of $10^{-9}$. We restarted the BI-CGSTAB every 5 steps.

Tables 1 - 4 illustrate the numerical behavior of the scattered field at arbitrary far-field points, generated by the potential $V$ defined in (3); the incident field is produced by a single point source. In Tables 1 and 2 , we set the potential $V(x, y)=e^{-40\left(x^{2}+y^{2}\right)}$. Tables 3 and 4 illustrate the numerical behavior of the scattered field, generated by a model of the human skull, as shown in Figures 2 and 3. The headings of the Tables are as follows:

$k$ is the wave number defined in (2);

the computational grid is $N \times N$ for a total of $N^{2}$ discretization points;

the computational grid is size $e_{o b j}$ wavelengths $\times$ size $_{o b j}$ wavelengths;

$N_{\lambda}$ is the number of discretization points per wavelength; 


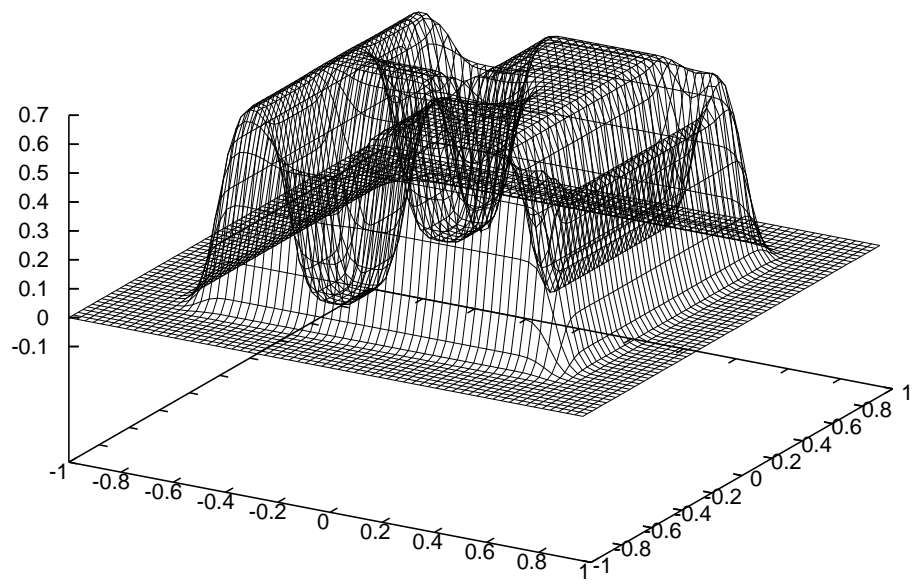

Figure 2: The human skull model

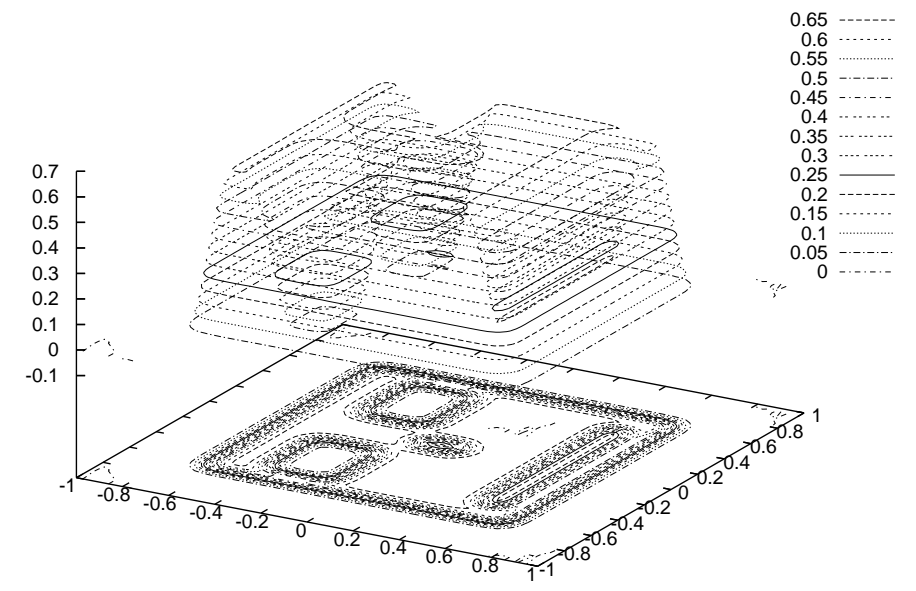

Figure 3: The human skull model viewed from the top

Table 1: $10^{\text {th }}$ order convergence of the algorithm for Gaussian objects

\begin{tabular}{|c|c|c|c|c|c|c|}
\hline$k$ & $N$ & size $_{\text {obj }}$ & $N_{\lambda}$ & $E_{\text {rel }}$ & $N_{\text {iter }}$ & $t_{C P U}$ \\
\hline 25 & 50 & $8 \lambda$ & 6.28 & $6.33 \mathrm{E}-06$ & 16 & $1.2 \mathrm{E}-01$ \\
25 & 100 & $8 \lambda$ & 12.6 & $6.63 \mathrm{E}-09$ & 16 & $5.9 \mathrm{E}-01$ \\
25 & 200 & $8 \lambda$ & 25.1 & $6.04 \mathrm{E}-12$ & 16 & $2.6 \mathrm{E}+00$ \\
25 & 400 & $8 \lambda$ & 50.2 & $7.25 \mathrm{E}-13$ & 16 & $1.1 \mathrm{E}+01$ \\
25 & 800 & $8 \lambda$ & 100 & $6.32 \mathrm{E}-13$ & 16 & $5.5 \mathrm{E}+01$ \\
25 & 1600 & $8 \lambda$ & 201 & - & 16 & $2.4 \mathrm{E}+02$ \\
\hline
\end{tabular}


Table 2: Gaussian objects with a fixed number of points per wavelength

\begin{tabular}{|c|c|c|c|c|c|c|}
\hline$k$ & $N$ & size $_{\text {obj }}$ & $N_{\lambda}$ & $E_{\text {rel }}$ & $N_{\text {iter }}$ & $t_{C P U}$ \\
\hline 25 & 50 & $8 \lambda$ & 6.28 & $6.33 \mathrm{E}-06$ & 14 & $1.1 \mathrm{E}-01$ \\
50 & 100 & $16 \lambda$ & 6.28 & $3.80 \mathrm{E}-06$ & 20 & $7.2 \mathrm{E}-01$ \\
100 & 200 & $32 \lambda$ & 6.28 & $4.44 \mathrm{E}-06$ & 33 & $5.2 \mathrm{E}+00$ \\
200 & 400 & $64 \lambda$ & 6.28 & $8.26 \mathrm{E}-06$ & 61 & $4.4 \mathrm{E}+01$ \\
400 & 800 & $128 \lambda$ & 6.28 & $1.60 \mathrm{E}-05$ & 171 & $6.2 \mathrm{E}+02$ \\
800 & 1600 & $255 \lambda$ & 6.28 & - & 891 & $1.4 \mathrm{E}+04$ \\
\hline
\end{tabular}

Table 3: $10^{\text {th }}$ order convergence of the algorithm for the simulated human skull

\begin{tabular}{|c|c|c|c|c|c|c|}
\hline$k$ & $N$ & size $_{\text {obj }}$ & $N_{\lambda}$ & $E_{\text {rel }}$ & $N_{\text {iter }}$ & $t_{C P U}$ \\
\hline 25 & 50 & $8 \lambda$ & 6.28 & $1.18 \mathrm{E}-04$ & 134 & $1.1 \mathrm{E}+00$ \\
25 & 100 & $8 \lambda$ & 12.6 & $1.91 \mathrm{E}-07$ & 133 & $4.7 \mathrm{E}+00$ \\
25 & 200 & $8 \lambda$ & 25.1 & $2.05 \mathrm{E}-10$ & 134 & $2.1 \mathrm{E}+01$ \\
25 & 400 & $8 \lambda$ & 50.2 & $4.56 \mathrm{E}-12$ & 135 & $9.7 \mathrm{E}+01$ \\
25 & 800 & $8 \lambda$ & 100 & $7.55 \mathrm{E}-12$ & 132 & $4.7 \mathrm{E}+02$ \\
25 & 1600 & $8 \lambda$ & 201 & - & 132 & $2.0 \mathrm{E}+03$ \\
\hline
\end{tabular}

Table 4: The simulated human skull with a fixed number of points per wavelength

\begin{tabular}{|c|c|c|c|c|c|c|}
\hline$k$ & $N$ & size $_{\text {obj }}$ & $N_{\lambda}$ & $E_{\text {rel }}$ & $N_{\text {iter }}$ & $t_{C P U}$ \\
\hline 25 & 50 & $8 \lambda$ & 6.28 & $1.17 \mathrm{E}-04$ & 97 & $7.6 \mathrm{E}-01$ \\
50 & 100 & $16 \lambda$ & 6.28 & $1.55 \mathrm{E}-05$ & 165 & $5.8 \mathrm{E}+00$ \\
100 & 200 & $32 \lambda$ & 6.28 & $1.03 \mathrm{E}-05$ & 328 & $5.2 \mathrm{E}+01$ \\
200 & 400 & $64 \lambda$ & 6.28 & $1.69 \mathrm{E}-05$ & 756 & $5.5 \mathrm{E}+02$ \\
400 & 800 & $128 \lambda$ & 6.28 & $2.21 \mathrm{E}-05$ & 3286 & $1.2 \mathrm{E}+04$ \\
800 & 1600 & $255 \lambda$ & 6.28 & - & 13568 & $2.1 \mathrm{E}+05$ \\
\hline
\end{tabular}


$E_{r e l}$ is the average of the relative errors of the solution for the scattered field at twenty chosen far-field points;

$N_{i t e r}$ is the number of iterations used by the BI-CGSTAB;

$t_{C P U}$ is the $\mathrm{CPU}$ time required in seconds.

The following observations can be made from the tables above, and from the more detailed numerical tests performed by the authors.

1. For smooth scattering objects, the numerical algorithm of Section 4 displays $10^{\text {th }}$ order convergence; the CPU time required to obtain requested precision is proportional to $N_{\text {iter }} \cdot N^{2} \log N$, where $N^{2}$ is the total number of discretization points, $N_{i t e r}$ is determined by the requested precision, the number of iterations before restarting the iterative solver, the size and the structure of the scattering objects.

2. For sufficiently smooth scatterers, the relative precision of the solution is determined by the number of discretization points per wavelength. For example, to obtain 5-digit precision, we need roughly 6.5 points per wavelength. Thus, with our constraint of 2 GB RAM, five digits can be obtained for scattering objects as large as 300 wavelengths $\times 300$ wavelengths.

3. The number of iterations increases dramatically as the size of the scattering object increases, as shown in Tables 2, 4.

\section{Conclusions}

In this paper, we construct an iterative algorithm for the solution of two-dimensional forward scattering problems. The scheme is based on the combination of high-order quadrature formulae, rapid numerical application of the integral operator in the Lippmann-Schwinger equation, and the stabilized bi-conjugate gradient method (BI-CGSTAB). As illustrated via several numerical examples, the scheme is $(2 p+4)^{t h}(p=0,1,2,3, \ldots)$ order convergent; the computational complexity of the algorithm is $O\left(N_{i t e r} \cdot N^{2} \log N\right)$, where $N_{\text {iter }}$ is the number of iterations used by the iterative solver, and $N^{2}$ is the total number of discretization points.

The approach we use for the design of high order center-corrected quadrature formulae introduced in this paper is not limited to functions of the form (13) in two dimensions; it is also applicable to functions of the form (14) in three dimensions, as well as many similar situations. Furthermore, the method does not require access to each of the functions $P, Q$ separately in (13) and (14); it only requires the evaluation of the whole kernel $K$ given a pair of points $(x, y)$. Quadrature formulae of order higher than 10 can also be constructed, though the derivations become more tedious. Finally, the scheme is easily extended to rectangular regions of the form $[-a, a] \times[-b, b]$, even though this paper only discusses on the square region $[-a, a] \times[-a, a]$.

Acknowledgments. The authors would like to thank Mark Tygert for helpful discussions. 


\section{Appendix A}

Lemmas $6.1-6.5$ below provide analytical formulae for

$$
\int_{-\infty}^{\infty} \int_{-\infty}^{\infty}\left(x^{i-1} y^{j-1} H_{0}\left(k \sqrt{x^{2}+y^{2}}\right)\right) d x d y-\sum_{(p, q) \neq(0,0)}\left((p h)^{i-1}(q h)^{j-1} H_{0}\left(k \sqrt{(p h)^{2}+(q h)^{2}}\right)\right) \cdot h^{2}
$$

with $(i, j)=\{(3,1),(5,1),(3,3),(7,1),(5,3)\}$. The proofs are straightforward and tedious, and use the help of Mathematica.

Lemma 6.1 For any $k \in \mathbb{C}^{+}$and $h>0$,

$$
\begin{aligned}
& D_{1}=\int_{-\infty}^{\infty} \int_{-\infty}^{\infty} H_{0}^{(1)}(k r) x^{2} d x d y-\sum_{(p, q) \neq(0,0)} H_{0}^{(1)}\left(k \sqrt{(p h)^{2}+(q h)^{2}}\right) \cdot(p h)^{2} \cdot h^{2} \\
& =\frac{4}{\pi} \int_{-\infty}^{\infty} \frac{d \lambda}{\sqrt{k^{2}-\lambda^{2}}} \cdot\left(\frac{2}{\left(i \alpha_{1}\right)^{3}\left(i \alpha_{2}\right)}-\frac{h^{4}}{2} \cdot \frac{e^{i \alpha_{1} h}\left(e^{i \alpha_{1} h}+1\right)\left(e^{i \alpha_{2} h}+1\right)}{\left(e^{i \alpha_{1} h}-1\right)^{3}\left(e^{i \alpha_{2} h}-1\right)}\right) \\
& =\frac{4}{\pi} \int_{-\infty}^{\infty} \frac{d \lambda}{\sqrt{k^{2}-\lambda^{2}}} \cdot \frac{2}{\alpha_{1}^{3} \alpha_{2}\left(1+x_{1}\right)^{3}\left(1+x_{2}\right)} \\
& \cdot\left(\frac{\left(\alpha_{2} h\right)^{2}}{12}+3 z_{1}+z_{2}+3 y_{1}^{2}+\frac{3}{2} y_{1}\left(i \alpha_{1} h\right)+3 y_{1} y_{2}+\frac{3}{2} y_{1}\left(i \alpha_{2} h\right)+\frac{3}{2} y_{2}\left(i \alpha_{1} h\right)\right. \\
& +x_{1}^{3}+3 x_{1}^{2} x_{2}+x_{1}^{3} x_{2}-\frac{1}{2} i \alpha_{2} h y_{2}+\frac{1}{2} \alpha_{1}^{2} h^{2}\left(x_{1}^{2}+2 x_{1}\right) \\
& \left.+\frac{3}{4} \alpha_{1} \alpha_{2} h^{2}\left(x_{1} x_{2}+x_{1}+x_{2}\right)+\frac{1}{4} i \alpha_{1}^{2} \alpha_{2} h^{3}\left(1+x_{1}\right)^{2}\left(1+x_{2}\right)\right)
\end{aligned}
$$

where $r, \alpha_{1}, \alpha_{2}, x_{1}, x_{2}, y_{1}, y_{2}, z_{1}, z_{2}$ are defined by (88), (89), and (93)-(95). 
Lemma 6.2 For any $k \in \mathbb{C}^{+}$and $h>0$,

$$
\begin{aligned}
& D_{2}=\int_{-\infty}^{\infty} \int_{-\infty}^{\infty} H_{0}^{(1)}(k r) x^{4} d x d y-\sum_{(p, q) \neq(0,0)} H_{0}^{(1)}\left(k \sqrt{(p h)^{2}+(q h)^{2}}\right) \cdot(p h)^{4} \cdot h^{2} \\
& =\frac{4}{\pi} \int_{-\infty}^{\infty} \frac{d \lambda}{\sqrt{k^{2}-\lambda^{2}}} \\
& \cdot\left(\frac{24}{\left(i \alpha_{1}\right)^{5}\left(i \alpha_{2}\right)}-\frac{h^{6}}{2} \cdot \frac{e^{i \alpha_{1} h}+11 e^{2 i \alpha_{1} h}+11 e^{3 i \alpha_{1} h}+e^{4 i \alpha_{1} h}}{\left(e^{i \alpha_{1} h}-1\right)^{5}} \cdot \frac{e^{i \alpha_{2} h}+1}{e^{i \alpha_{2} h}-1}\right) \\
& =\frac{4}{\pi} \int_{-\infty}^{\infty} \frac{d \lambda}{\sqrt{k^{2}-\lambda^{2}}} \cdot \frac{1}{\alpha_{1}^{5} \alpha_{2}\left(1+x_{1}\right)^{5}\left(1+x_{2}\right)} \\
& \cdot\left(2\left(i \alpha_{2} h\right)^{2}+\left(i \alpha_{2} h\right)^{3}+5\left(i \alpha_{1} h\right)\left(i \alpha_{2} h\right)^{2}+\frac{3}{10}\left(i \alpha_{2} h\right)^{4}+\frac{20}{3}\left(i \alpha_{1} h\right)^{2}\left(i \alpha_{2} h\right)^{2}\right. \\
& +\frac{5}{2}\left(i \alpha_{1} h\right)\left(i \alpha_{2} h\right)^{3}+\frac{1}{2}\left(i \alpha_{1} h\right)^{4}\left(i \alpha_{2} h\right)+4 x_{1}\left(i \alpha_{1} h\right)^{4}+\frac{45}{2} x_{1}\left(i \alpha_{1} h\right)^{3}\left(i \alpha_{2} h\right) \\
& +2 x_{1}\left(i \alpha_{1} h\right)^{4}\left(i \alpha_{2} h\right)+45 x_{1}^{2}\left(i \alpha_{1} h\right)^{3}+25 x_{1}^{2}\left(i \alpha_{1} h\right)^{2}\left(i \alpha_{2} h\right)+6 x_{1}^{2}\left(i \alpha_{1} h\right)^{4} \\
& +\frac{45}{2} x_{1}^{2}\left(i \alpha_{1} h\right)^{3}\left(i \alpha_{2} h\right)+3 x_{1}^{2}\left(i \alpha_{1} h\right)^{4}\left(i \alpha_{2} h\right)+15 x_{1}^{3}\left(i \alpha_{1} h\right)^{3}+4 x_{1}^{3}\left(i \alpha_{1} h\right)^{4} \\
& +\frac{15}{2} x_{1}^{3}\left(i \alpha_{1} h\right)^{3}\left(i \alpha_{2} h\right)+2 x_{1}^{3}\left(i \alpha_{1} h\right)^{4}\left(i \alpha_{2} h\right)+x_{1}^{4}\left(i \alpha_{1} h\right)^{4}+\frac{1}{2} x_{1}^{4}\left(i \alpha_{1} h\right)^{4}\left(i \alpha_{2} h\right) \\
& +\frac{15}{2} x_{2}\left(i \alpha_{1} h\right)^{3}\left(i \alpha_{2} h\right)+\frac{1}{2} x_{2}\left(i \alpha_{1} h\right)^{4}\left(i \alpha_{2} h\right)+50 x_{1} x_{2}\left(i \alpha_{1} h\right)^{2}\left(i \alpha_{2} h\right) \\
& +\frac{45}{2} x_{1} x_{2}\left(i \alpha_{1} h\right)^{3}\left(i \alpha_{2} h\right)+2 x_{1} x_{2}\left(i \alpha_{1} h\right)^{4}\left(i \alpha_{2} h\right)+25 x_{1}^{2} x_{2}\left(i \alpha_{1} h\right)^{2}\left(i \alpha_{2} h\right) \\
& +\frac{45}{2} x_{1}^{2} x_{2}\left(i \alpha_{1} h\right)^{3}\left(i \alpha_{2} h\right)+3 x_{1}^{2} x_{2}\left(i \alpha_{1} h\right)^{4}\left(i \alpha_{2} h\right)+\frac{15}{2} x_{1}^{3} x_{2}\left(i \alpha_{1} h\right)^{3}\left(i \alpha_{2} h\right) \\
& +2 x_{1}^{3} x_{2}\left(i \alpha_{1} h\right)^{4}\left(i \alpha_{2} h\right)+\frac{1}{2} x_{1}^{4} x_{2}\left(i \alpha_{1} h\right)^{4}\left(i \alpha_{2} h\right)+12 w_{2}\left(i \alpha_{2} h\right)+30 z_{2}\left(i \alpha_{1} h\right)\left(i \alpha_{2} h\right) \\
& +40 y_{2}\left(i \alpha_{1} h\right)^{2}\left(i \alpha_{2} h\right)+15 y_{1}\left(i \alpha_{1} h\right)\left(i \alpha_{2} h\right)^{2}+30 y_{1} y_{2}\left(i \alpha_{1} h\right)\left(i \alpha_{2} h\right)-120 u_{1}-24 u_{2} \\
& -180 w_{1}\left(i \alpha_{1} h\right)-60 w_{2}\left(i \alpha_{1} h\right)-60 w_{1}\left(i \alpha_{2} h\right)-160 z_{1}\left(i \alpha_{1} h\right)^{2}-90 z_{1}\left(i \alpha_{1} h\right)\left(i \alpha_{2} h\right) \\
& -80 z_{2}\left(i \alpha_{1} h\right)^{2}+35 y_{1}\left(i \alpha_{1} h\right)^{3}-40 y_{1}\left(i \alpha_{1} h\right)^{2}\left(i \alpha_{2} h\right)-130 y_{1}^{2}\left(i \alpha_{1} h\right)^{2} \\
& -180 y_{1}^{2}\left(i \alpha_{1} h\right)\left(i \alpha_{2} h\right)-240 y_{1}^{3}\left(i \alpha_{1} h\right)-120 y_{1}^{3}\left(i \alpha_{2} h\right)-120 y_{1}^{4}-30 y_{2}\left(i \alpha_{1} h\right)^{3} \\
& -180 y_{1} y_{2}\left(i \alpha_{1} h\right)^{2}-360 y_{1}^{2} y_{2}\left(i \alpha_{1} h\right)-240 y_{1}^{3} y_{2}-20 z_{1}\left(i \alpha_{2} h\right)^{2}-240 z_{1}^{2}-120 z_{1} z_{2} \\
& -360 y_{1}^{2}\left(i \alpha_{1} h\right)-240 y_{1}^{3}-120 y_{1}^{2}\left(i \alpha_{2} h\right)-240 y_{1} y_{2}\left(i \alpha_{1} h\right)-240 y_{1}^{2} y_{2} \\
& \left.-24 x_{1}^{5}-120 x_{1}^{4} x_{2}-24 x_{1}^{5} x_{2}\right)
\end{aligned}
$$

where

$$
\begin{aligned}
& w_{1}=z_{1}-\frac{\left(i \alpha_{1} h\right)^{3}}{24}=\sum_{n=4}^{\infty} \frac{\left(i \alpha_{1} h\right)^{n}}{(n+1) !}, w_{2}=z_{2}-\frac{\left(i \alpha_{2} h\right)^{3}}{24}=\sum_{n=4}^{\infty} \frac{\left(i \alpha_{2} h\right)^{n}}{(n+1) !}, \\
& u_{1}=w_{1}-\frac{\left(i \alpha_{1} h\right)^{4}}{120}=\sum_{n=5}^{\infty} \frac{\left(i \alpha_{1} h\right)^{n}}{(n+1) !}, u_{2}=w_{2}-\frac{\left(i \alpha_{2} h\right)^{4}}{120}=\sum_{n=5}^{\infty} \frac{\left(i \alpha_{2} h\right)^{n}}{(n+1) !},
\end{aligned}
$$

and $r, \alpha_{1}, \alpha_{2}, x_{1}, x_{2}, y_{1}, y_{2}, z_{1}, z_{2}$ are defined by (88), (89) and (93)-(95). 
Lemma 6.3 For any $k \in \mathbb{C}^{+}$and $h>0$,

$$
\begin{aligned}
& D_{3}=\int_{-\infty}^{\infty} \int_{-\infty}^{\infty} H_{0}^{(1)}(k r) x^{2} y^{2} d x d y-\sum_{(p, q) \neq(0,0)} H_{0}^{(1)}\left(k \sqrt{(p h)^{2}+(q h)^{2}}\right) \cdot(p h)^{2}(q h)^{2} \cdot h^{2} \\
& =\frac{4}{\pi} \int_{-\infty}^{\infty} \frac{d \lambda}{\sqrt{k^{2}-\lambda^{2}}}\left(\frac{4}{\left(i \alpha_{1}\right)^{3}\left(i \alpha_{2}\right)^{3}}-h^{6} \cdot \frac{e^{i \alpha_{1} h}\left(e^{i \alpha_{1} h}+1\right)}{\left(e^{i \alpha_{1} h}-1\right)^{3}} \cdot \frac{e^{i \alpha_{1} h}\left(e^{i \alpha_{1} h}+1\right)}{\left(e^{i \alpha_{1} h}-1\right)^{3}}\right) \\
& =\frac{4}{\pi} \int_{-\infty}^{\infty} \frac{d \lambda}{\sqrt{k^{2}-\lambda^{2}}} \cdot \frac{-4}{\alpha_{1}^{3} \alpha_{2}^{3}\left(1+x_{1}\right)^{3}\left(1+x_{2}\right)^{3}} \\
& \cdot\left(\frac{1}{240}\left(i \alpha_{1} h\right)^{4}+\frac{1}{240}\left(i \alpha_{2} h\right)^{4}+\frac{3}{4} z_{1}\left(i \alpha_{1} h\right)^{2}+\frac{3}{4} z_{2}\left(i \alpha_{2} h\right)^{2}\right. \\
& +\frac{9}{2}\left(i \alpha_{1} h\right)\left(i \alpha_{2} h\right)\left(z_{1}+z_{2}\right)+\frac{15}{4} z_{1}\left(i \alpha_{2} h\right)^{2}+\frac{15}{4} z_{2}\left(i \alpha_{1} h\right)^{2}-\frac{3}{2} y_{1}\left(i \alpha_{1} h\right)^{2}\left(i \alpha_{2} h\right) \\
& -\frac{3}{2} y_{2}\left(i \alpha_{1} h\right)\left(i \alpha_{2} h\right)^{2}+3 u_{1}+3 u_{2}+\frac{9}{2} w_{2}\left(i \alpha_{1} h\right)+\frac{9}{2} w_{1}\left(i \alpha_{2} h\right) \\
& +\frac{3}{2} w_{1}\left(i \alpha_{1} h\right)+\frac{3}{2} w_{2}\left(i \alpha_{2} h\right)-\frac{9}{4}\left(i \alpha_{1} h\right)\left(i \alpha_{2} h\right)\left(z_{1}+z_{2}\right)+3 z_{1}^{2}+3 z_{2}^{2} \\
& +9 z_{1} z_{2}-\frac{1}{2} y_{1}\left(i \alpha_{1} h\right)^{3}-\frac{1}{2} y_{2}\left(i \alpha_{2} h\right)^{3}-\frac{1}{2} y_{1}^{2}\left(i \alpha_{1} h\right)^{2}-\frac{1}{2} y_{2}^{2}\left(i \alpha_{2} h\right)^{2} \\
& +\frac{9}{4}\left(i \alpha_{1} h\right)\left(i \alpha_{2} h\right)\left(3 y_{1} y_{2}+\frac{1}{6} y_{1}\left(i \alpha_{2} h\right)+\frac{1}{6} y_{2}\left(i \alpha_{1} h\right)\right)+\frac{9}{8} y_{1}\left(i \alpha_{1} h\right)^{2}\left(i \alpha_{2} h\right) \\
& +\frac{3}{8} y_{1}\left(i \alpha_{2} h\right)^{3}+\frac{9}{4} y_{1}^{2}\left(i \alpha_{1} h\right)\left(i \alpha_{2} h\right)+\frac{9}{4} y_{1}^{2}\left(i \alpha_{2} h\right)^{2}+\frac{3}{2} y_{1}^{3}\left(i \alpha_{2} h\right) \\
& +\frac{3}{8} y_{2}\left(i \alpha_{1} h\right)^{3}+\frac{9}{8} y_{2}\left(i \alpha_{1} h\right)\left(i \alpha_{2} h\right)^{2}+\frac{9}{4} y_{1} y_{2}\left(i \alpha_{1} h\right)^{2}+\frac{9}{4} y_{1} y_{2}\left(i \alpha_{2} h\right)^{2} \\
& +\frac{9}{2} y_{1}^{2} y_{2}\left(i \alpha_{1} h\right)+9 y_{1}^{2} y_{2}\left(i \alpha_{2} h\right)+3 y_{1}^{3} y_{2}+\frac{9}{4} y_{2}^{2}\left(i \alpha_{1} h\right)^{2}+\frac{9}{4} y_{2}^{2}\left(i \alpha_{1} h\right)\left(i \alpha_{2} h\right) \\
& +9 y_{1} y_{2}^{2}\left(i \alpha_{1} h\right)+\frac{9}{2} y_{1} y_{2}^{2}\left(i \alpha_{2} h\right)+9 y_{1}^{2} y_{2}^{2}+\frac{3}{2} y_{2}^{3}\left(i \alpha_{1} h\right)+3 y_{1} y_{2}^{3} \\
& -\frac{1}{4}\left(i \alpha_{1} h\right)^{2}\left(i \alpha_{2} h\right)^{2}\left(2 x_{1}+x_{1}^{2}+2 x_{2}+4 x_{1} x_{2}+2 x_{1}^{2} x_{2}+x_{2}^{2}\right. \\
& \left.+2 x_{1} x_{2}^{2}+x_{1}^{2} x_{2}^{2}\right)+\frac{3}{2} y_{1}^{2}\left(i \alpha_{1} h\right)+y_{1}^{3}+\frac{3}{2} y_{2}^{2}\left(i \alpha_{2} h\right)+y_{2}^{3}+\frac{9}{2} y_{1}^{2}\left(i \alpha_{2} h\right) \\
& +9 y_{1} y_{2}\left(i \alpha_{1} h+i \alpha_{2} h\right)+9 y_{1}^{2} y_{2}+\frac{9}{2} y_{2}^{2}\left(i \alpha_{1} h\right)+9 y_{1} y_{2}^{2} \\
& -\frac{3}{4}\left(i \alpha_{1} h\right)^{2}\left(i \alpha_{2} h\right)\left(x_{1}^{2}+2 x_{1} x_{2}+x_{1}^{2} x_{2}\right) \\
& \left.-\frac{3}{4}\left(i \alpha_{1} h\right)\left(i \alpha_{2} h\right)^{2}\left(x_{2}^{2}+2 x_{1} x_{2}+x_{1} x_{2}^{2}\right)+3 x_{1}^{3} x_{2}^{2}+3 x_{1}^{2} x_{2}^{3}+x_{1}^{3} x_{2}^{3}\right),
\end{aligned}
$$

where $r, \alpha_{1}, \alpha_{2}, x_{1}, x_{2}, y_{1}, y_{2}, z_{1}, z_{2}, w_{1}, w_{2}, u_{1}, u_{2}$ are defined by (88), (89), (93)-(95), (127) and (128). 
Lemma 6.4 For any $k \in \mathbb{C}^{+}$and $h>0$,

$$
\begin{aligned}
& D_{4}=\int_{-\infty}^{\infty} \int_{-\infty}^{\infty} H_{0}^{(1)}(k r) x^{6} d x d y-\sum_{(p, q) \neq(0,0)} H_{0}^{(1)}\left(k \sqrt{(p h)^{2}+(q h)^{2}}\right) \cdot(p h)^{6} \cdot h^{2} \\
& =\frac{4}{\pi} \int_{-\infty}^{\infty} \frac{d \lambda}{\sqrt{k^{2}-\lambda^{2}}} \\
& \cdot\left(\frac{720}{\alpha_{1}^{7} \alpha_{2}}-\frac{h^{8}}{2} \cdot \frac{e^{i \alpha_{1} h}+57 e^{2 i \alpha_{1} h}+302 e^{3 i \alpha_{1} h}+302 e^{4 i \alpha_{1} h}+57 e^{5 i \alpha_{1} h}+e^{6 i \alpha_{1} h}}{\left(e^{i \alpha_{1} h}-1\right)^{7}} \cdot \frac{e^{i \alpha_{2} h}+1}{e^{i \alpha_{2} h}-1}\right) \\
& =\frac{4}{\pi} \int_{-\infty}^{\infty} \frac{d \lambda}{\sqrt{k^{2}-\lambda^{2}}} \cdot \frac{1}{\alpha_{1}^{7} \alpha_{2}\left(1+x_{1}\right)^{7}\left(1+x_{2}\right)} \cdot(A+B),
\end{aligned}
$$

where

$$
\begin{aligned}
& A=60\left(\alpha_{2} h\right)^{2}-30\left(i \alpha_{2} h\right)^{3}-210\left(i \alpha_{1} h\right)\left(i \alpha_{2} h\right)^{2}-9\left(\alpha_{2} h\right)^{4}-105\left(\alpha_{1} h\right)\left(\alpha_{2} h\right)^{3} \\
& -385\left(\alpha_{1} h\right)^{2}\left(\alpha_{2} h\right)^{2}+37800 y_{1}^{2}\left(i \alpha_{1} h\right)+25200 y_{1}^{3}+7560 y_{1}^{2}\left(i \alpha_{2} h\right) \\
& +15120 y_{1} y_{2}\left(i \alpha_{1} h\right)+15120 y_{1}^{2} y_{2}+15120 x_{1}^{5}+5040 x_{1}^{6}+720 x_{1}^{7}+25200 x_{1}^{4} x_{2} \\
& +15120 x_{1}^{5} x_{2}+5040 x_{1}^{6} x_{2}+720 x_{1}^{7} x_{2}+5040 u_{1}+720 u_{2}+12600 w_{1}\left(i \alpha_{1} h\right) \\
& -360 w_{2}\left(i \alpha_{2} h\right)+2520 w_{2}\left(i \alpha_{1} h\right)+2520 w_{1}\left(i \alpha_{2} h\right)-17220\left(\alpha_{1} h\right)^{2} z_{1} \\
& +6300 z_{1}\left(i \alpha_{1} h\right)\left(i \alpha_{2} h\right)+1260 z_{2}\left(\alpha_{1} h\right)\left(\alpha_{2} h\right)-4620\left(\alpha_{1} h\right)^{2} z_{2}+15120 z_{1}^{2} \\
& +2940 y_{1}\left(i \alpha_{1} h\right)^{3}-6090 y_{1}\left(\alpha_{1} h\right)^{2}\left(i \alpha_{2} h\right)-34440 y_{1}^{2}\left(\alpha_{1} h\right)^{2}-18900 y_{1}^{2}\left(\alpha_{1} h\right)\left(\alpha_{2} h\right) \\
& +50400 y_{1}^{3}\left(i \alpha_{1} h\right)+12600 y_{1}^{3}\left(i \alpha_{2} h\right)+25200 y_{1}^{4}+3150 y_{2}\left(i \alpha_{1} h\right)^{3} \\
& -18900 y_{1} y_{2}\left(\alpha_{1} h\right)^{2}+37800 y_{1}^{2} y_{2}\left(i \alpha_{1} h\right)+25200 y_{1}^{3} y_{2}-840 z_{1}\left(\alpha_{2} h\right)^{2}+5040 z_{1} z_{2} \\
& +2310 y_{2}\left(\alpha_{1} h\right)^{2}\left(i \alpha_{2} h\right)+630 y_{1}\left(i \alpha_{1} h\right)\left(\alpha_{2} h\right)^{2}+1260 y_{1} y_{2}\left(\alpha_{1} h\right)\left(\alpha_{2} h\right) \\
& -63\left(i \alpha_{1} h\right)^{5}-301\left(i \alpha_{1} h\right)^{4}\left(i \alpha_{2} h\right)+\left(\alpha_{1} h\right)^{6}+\frac{63}{2}\left(\alpha_{1} h\right)^{5}\left(\alpha_{2} h\right)+\frac{1}{2}\left(\alpha_{1} h\right)^{6}\left(i \alpha_{2} h\right) \\
& -2408 x_{1}\left(\alpha_{1} h\right)^{4}-3150 x_{1}\left(\alpha_{1} h\right)^{3}\left(\alpha_{2} h\right)-315 x_{1}\left(i \alpha_{1} h\right)^{5}-1204 x_{1}\left(i \alpha_{1} h\right)^{4}\left(i \alpha_{2} h\right) \\
& +6 x_{1}\left(\alpha_{1} h\right)^{6}+\frac{315}{2} x_{1}\left(\alpha_{1} h\right)^{5}\left(\alpha_{2} h\right)+3 x_{1}\left(\alpha_{1} h\right)^{6}\left(i \alpha_{2} h\right)-6300 x_{1}^{2}\left(i \alpha_{1} h\right)^{3} \\
& +1680 x_{1}^{2}\left(\alpha_{1} h\right)^{2}\left(i \alpha_{2} h\right)-3612 x_{1}^{2}\left(\alpha_{1} h\right)^{4}-3150 x_{1}^{2}\left(\alpha_{1} h\right)^{3}\left(\alpha_{2} h\right)-630 x_{1}^{2}\left(i \alpha_{1} h\right)^{5} \\
& -1806 x_{1}^{2}\left(i \alpha_{1} h\right)^{4}\left(i \alpha_{2} h\right)+15 x_{1}^{2}\left(\alpha_{1} h\right)^{6}+315 x_{1}^{2}\left(\alpha_{1} h\right)^{5}\left(\alpha_{2} h\right)+\frac{15}{2} x_{1}^{2}\left(\alpha_{1} h\right)^{6}\left(i \alpha_{2} h\right) \\
& -2100 x_{1}^{3}\left(i \alpha_{1} h\right)^{3}-2408 x_{1}^{3}\left(\alpha_{1} h\right)^{4}-1050 x_{1}^{3}\left(\alpha_{1} h\right)^{3}\left(\alpha_{2} h\right)-630 x_{1}^{3}\left(i \alpha_{1} h\right)^{5} \\
& -1204 x_{1}^{3}\left(i \alpha_{1} h\right)^{4}\left(i \alpha_{2} h\right)+20 x_{1}^{3}\left(\alpha_{1} h\right)^{6}+315 x_{1}^{3}\left(\alpha_{1} h\right)^{5}\left(\alpha_{2} h\right)+10 x_{1}^{3}\left(\alpha_{1} h\right)^{6}\left(i \alpha_{2} h\right) \\
& -602 x_{1}^{4}\left(\alpha_{1} h\right)^{4}-315 x_{1}^{4}\left(i \alpha_{1} h\right)^{5}-301 x_{1}^{4}\left(i \alpha_{1} h\right)^{4}\left(i \alpha_{2} h\right)+15 x_{1}^{4}\left(\alpha_{1} h\right)^{6} \\
& +\frac{315}{2} x_{1}^{4}\left(\alpha_{1} h\right)^{5}\left(\alpha_{2} h\right)+\frac{15}{2} x_{1}^{4}\left(\alpha_{1} h\right)^{6}\left(i \alpha_{2} h\right)-63 x_{1}^{5}\left(i \alpha_{1} h\right)^{5}+6 x_{1}^{5}\left(\alpha_{1} h\right)^{6} \\
& +\frac{63}{2} x_{1}^{5}\left(\alpha_{1} h\right)^{5}\left(\alpha_{2} h\right)+3 x_{1}^{5}\left(\alpha_{1} h\right)^{6}\left(i \alpha_{2} h\right)+x_{1}^{6}\left(\alpha_{1} h\right)^{6}+\frac{1}{2} x_{1}^{6}\left(\alpha_{1} h\right)^{6}\left(i \alpha_{2} h\right),
\end{aligned}
$$




$$
\begin{aligned}
& B=-1050 x_{2}\left(\alpha_{1} h\right)^{3}\left(\alpha_{2} h\right)-301 x_{2}\left(\alpha_{1} h\right)^{4}\left(i \alpha_{2} h\right)+\frac{63}{2} x_{2}\left(\alpha_{1} h\right)^{5}\left(\alpha_{2} h\right) \\
& +\frac{1}{2} x_{2}\left(\alpha_{1} h\right)^{6}\left(i \alpha_{2} h\right)+3360 x_{1} x_{2}\left(\alpha_{1} h\right)^{2}\left(i \alpha_{2} h\right)-3150 x_{1} x_{2}\left(\alpha_{1} h\right)^{3}\left(\alpha_{2} h\right) \\
& -1204 x_{1} x_{2}\left(\alpha_{1} h\right)^{4}\left(i \alpha_{2} h\right)+\frac{315}{2} x_{1} x_{2}\left(\alpha_{1} h\right)^{5}\left(\alpha_{2} h\right)+3 x_{1} x_{2}\left(\alpha_{1} h\right)^{6}\left(i \alpha_{2} h\right) \\
& +1680 x_{1}^{2} x_{2}\left(\alpha_{1} h\right)^{2}\left(i \alpha_{2} h\right)-3150 x_{1}^{2} x_{2}\left(\alpha_{1} h\right)^{3}\left(\alpha_{2} h\right)-1806 x_{1}^{2} x_{2}\left(\alpha_{1} h\right)^{4}\left(i \alpha_{2} h\right) \\
& +315 x_{1}^{2} x_{2}\left(\alpha_{1} h\right)^{5}\left(\alpha_{2} h\right)+\frac{15}{2} x_{1}^{2} x_{2}\left(\alpha_{1} h\right)^{6}\left(i \alpha_{2} h\right)-1050 x_{1}^{3} x_{2}\left(\alpha_{1} h\right)^{3}\left(\alpha_{2} h\right) \\
& -1204 x_{1}^{3} x_{2}\left(\alpha_{1} h\right)^{4}\left(i \alpha_{2} h\right)+315 x_{1}^{3} x_{2}\left(\alpha_{1} h\right)^{5}\left(\alpha_{2} h\right)+10 x_{1}^{3} x_{2}\left(\alpha_{1} h\right)^{6}\left(i \alpha_{2} h\right) \\
& -301 x_{1}^{4} x_{2}\left(\alpha_{1} h\right)^{4}\left(i \alpha_{2} h\right)+\frac{315}{2} x_{1}^{4} x_{2}\left(\alpha_{1} h\right)^{5}\left(\alpha_{2} h\right)+\frac{15}{2} x_{1}^{4} x_{2}\left(\alpha_{1} h\right)^{6}\left(i \alpha_{2} h\right) \\
& +\frac{63}{2} x_{1}^{5} x_{2}\left(\alpha_{1} h\right)^{5}\left(\alpha_{2} h\right)+3 x_{1}^{5} x_{2}\left(\alpha_{1} h\right)^{6}\left(i \alpha_{2} h\right)+\frac{1}{2} x_{1}^{6} x_{2}\left(\alpha_{1} h\right)^{6}\left(i \alpha_{2} h\right),
\end{aligned}
$$

and where $r, \alpha_{1}, \alpha_{2}, x_{1}, x_{2}, y_{1}, y_{2}, z_{1}, z_{2}, w_{1}, w_{2}, u_{1}, u_{2}$ are defined by (88), (89), (93)-(95), (127) and (128).

Lemma 6.5 For any $k \in \mathbb{C}^{+}$and $h>0$,

$$
\begin{aligned}
& D_{5}=\int_{-\infty}^{\infty} \int_{-\infty}^{\infty} H_{0}^{(1)}(k r) x^{4} y^{2} d x d y-\sum_{(p, q) \neq(0,0)} H_{0}^{(1)}\left(k \sqrt{(p h)^{2}+(q h)^{2}}\right) \cdot(p h)^{4}(q h)^{2} \cdot h^{2} \\
& =\frac{4}{\pi} \int_{-\infty}^{\infty} \frac{d \lambda}{\sqrt{k^{2}-\lambda^{2}}} \cdot\left(\frac{48}{\alpha_{1}^{5} \alpha_{2}^{3}}-h^{8} \cdot \frac{e^{i \alpha_{1} h}+11 e^{2 i \alpha_{1} h}+11 e^{3 i \alpha_{1} h}+e^{4 i \alpha_{1} h}}{\left(e^{i \alpha_{1} h}-1\right)^{5}} \cdot \frac{e^{i \alpha_{2} h} \cdot\left(e^{i \alpha_{2} h}+1\right)}{\left(e^{i \alpha_{2} h}-1\right)^{3}}\right) \\
& =\frac{4}{\pi} \int_{-\infty}^{\infty} \frac{d \lambda}{\sqrt{k^{2}-\lambda^{2}}} \cdot \frac{1}{\alpha_{1}^{5} \alpha_{2}^{3}\left(1+x_{1}\right)^{5}\left(1+x_{2}\right)^{3}} \cdot(C+D)
\end{aligned}
$$

where

$$
\begin{aligned}
& C=\frac{1}{5}\left(\alpha_{2} h\right)^{4}+240 u_{1}+360 w_{1}\left(i \alpha_{1} h\right)+144 u_{2}+360 w_{2}\left(i \alpha_{1} h\right) \\
& +360 w_{1}\left(i \alpha_{2} h\right)+72 w_{2}\left(i \alpha_{2} h\right)-320 z_{1}\left(\alpha_{1} h\right)^{2}-540 z_{1}\left(\alpha_{1} h\right)\left(\alpha_{2} h\right) \\
& -480 z_{2}\left(\alpha_{1} h\right)^{2}-300 z_{1}\left(\alpha_{2} h\right)^{2}-180 z_{2}\left(\alpha_{1} h\right)\left(\alpha_{2} h\right)-36 z_{2}\left(\alpha_{2} h\right)^{2}+480 z_{1}^{2} \\
& +720 z_{1} z_{2}+144 z_{2}^{2}-70 y_{1}\left(i \alpha_{1} h\right)^{3}-240 y_{1}\left(\alpha_{1} h\right)^{2}\left(i \alpha_{2} h\right)-210 y_{1}\left(i \alpha_{1} h\right)\left(\alpha_{2} h\right)^{2} \\
& +30 y_{1}\left(i \alpha_{2} h\right)^{3}-260 y_{1}^{2}\left(\alpha_{1} h\right)^{2}-1080 y_{1}^{2}\left(\alpha_{1} h\right)\left(\alpha_{2} h\right)-360 y_{1}^{2}\left(\alpha_{2} h\right)^{2} \\
& +480 y_{1}^{3}\left(i \alpha_{1} h\right)+720 y_{1}^{3}\left(i \alpha_{2} h\right)+240 y_{1}^{4}+180 y_{2}\left(i \alpha_{1} h\right)^{3}+120 y_{2}\left(i \alpha_{1} h\right)^{2}\left(i \alpha_{2} h\right) \\
& +30 y_{2}\left(i \alpha_{1} h\right)\left(\alpha_{2} h\right)^{2}-1080 y_{1} y_{2}\left(\alpha_{1} h\right)^{2}-1260 y_{1} y_{2}\left(\alpha_{1} h\right)\left(\alpha_{2} h\right) \\
& -180 y_{1} y_{2}\left(\alpha_{2} h\right)^{2}+2160 y_{1}^{2} y_{2}\left(i \alpha_{1} h\right)+1440 y_{1}^{2} y_{2}\left(i \alpha_{2} h\right)+1440 y_{1}^{3} y_{2}-360 y_{2}^{2}\left(\alpha_{1} h\right)^{2} \\
& -180 y_{2}^{2}\left(\alpha_{1} h\right)\left(\alpha_{2} h\right)+1440 y_{1} y_{2}^{2}\left(i \alpha_{1} h\right)+360 y_{1} y_{2}^{2}\left(i \alpha_{2} h\right)+1440 y_{1}^{2} y_{2}^{2}+120 y_{2}^{3}\left(i \alpha_{1} h\right) \\
& +240 y_{1} y_{2}^{3}+720 y_{1}^{2}\left(i \alpha_{1} h\right)+480 y_{1}^{3}+720 y_{1}^{2}\left(i \alpha_{2} h\right)+1440 y_{1} y_{2}\left(i \alpha_{1} h\right) \\
& +1440 y_{1}^{2} y_{2}+720 y_{1} y_{2}\left(i \alpha_{2} h\right)+360 y_{2}^{2}\left(i \alpha_{1} h\right)+720 y_{1} y_{2}^{2}+72 y_{2}^{2}\left(i \alpha_{2} h\right)+48 y_{2}^{3}+48 x_{1}^{5} \\
& +720 x_{1}^{4} x_{2}+144 x_{1}^{5} x_{2}+1440 x_{1}^{3} x_{2}^{2}+720 x_{1}^{4} x_{2}^{2}+144 x_{1}^{5} x_{2}^{2}+480 x_{1}^{2} x_{2}^{3}+480 x_{1}^{3} x_{2}^{3},
\end{aligned}
$$




$$
\begin{aligned}
& D=240 x_{1}^{4} x_{2}^{3}+48 x_{1}^{5} x_{2}^{3}-24 y_{2}\left(i \alpha_{2} h\right)^{3}+24 y_{2}^{2}\left(\alpha_{2} h\right)^{2}-3\left(i \alpha_{1} h\right)^{4}\left(i \alpha_{2} h\right) \\
& -15\left(i \alpha_{1} h\right)^{3}\left(i \alpha_{2} h\right)^{2}+\left(\alpha_{1} h\right)^{4}\left(\alpha_{2} h\right)^{2}-8 x_{1}\left(\alpha_{1} h\right)^{4}-135 x_{1}\left(\alpha_{1} h\right)^{3}\left(\alpha_{2} h\right) \\
& -100 x_{1}\left(\alpha_{1} h\right)^{2}\left(\alpha_{2} h\right)^{2}-12 x_{1}\left(\alpha_{1} h\right)^{4}\left(i \alpha_{2} h\right)-45 x_{1}\left(i \alpha_{1} h\right)^{3}\left(i \alpha_{2} h\right)^{2}+4 x_{1}\left(\alpha_{1} h\right)^{4}\left(\alpha_{2} h\right)^{2} \\
& -90 x_{1}^{2}\left(i \alpha_{1} h\right)^{3}-150 x_{1}^{2}\left(i \alpha_{1} h\right)^{2}\left(i \alpha_{2} h\right)-12 x_{1}^{2}\left(\alpha_{1} h\right)^{4}-135 x_{1}^{2}\left(\alpha_{1} h\right)^{3}\left(\alpha_{2} h\right) \\
& -50 x_{1}^{2}\left(\alpha_{1} h\right)^{2}\left(\alpha_{2} h\right)^{2}-18 x_{1}^{2}\left(\alpha_{1} h\right)^{4}\left(i \alpha_{2} h\right)-45 x_{1}^{2}\left(i \alpha_{1} h\right)^{3}\left(i \alpha_{2} h\right)^{2}+6 x_{1}^{2}\left(\alpha_{1} h\right)^{4}\left(\alpha_{2} h\right)^{2} \\
& -30 x_{1}^{3}\left(i \alpha_{1} h\right)^{3}-8 x_{1}^{3}\left(\alpha_{1} h\right)^{4}-45 x_{1}^{3}\left(\alpha_{1} h\right)^{3}\left(\alpha_{2} h\right)-12 x_{1}^{3}\left(\alpha_{1} h\right)^{4}\left(i \alpha_{2} h\right) \\
& -15 x_{1}^{3}\left(i \alpha_{1} h\right)^{3}\left(i \alpha_{2} h\right)^{2}+4 x_{1}^{3}\left(\alpha_{1} h\right)^{4}\left(\alpha_{2} h\right)^{2}-2 x_{1}^{4}\left(\alpha_{1} h\right)^{4}-3 x_{1}^{4}\left(\alpha_{1} h\right)^{4}\left(i \alpha_{2} h\right) \\
& +x_{1}^{4}\left(\alpha_{1} h\right)^{4}\left(\alpha_{2} h\right)^{2}-45 x_{2}\left(\alpha_{1} h\right)^{3}\left(\alpha_{2} h\right)-100 x_{2}\left(\alpha_{1} h\right)^{2}\left(\alpha_{2} h\right)^{2}-3 x_{2}\left(\alpha_{1} h\right)^{4}\left(i \alpha_{2} h\right) \\
& -30 x_{2}\left(i \alpha_{1} h\right)^{3}\left(i \alpha_{2} h\right)^{2}+2 x_{2}\left(\alpha_{1} h\right)^{4}\left(\alpha_{2} h\right)^{2}+300 x_{1} x_{2}\left(\alpha_{1} h\right)^{2}\left(i \alpha_{2} h\right) \\
& +120 x_{1} x_{2}\left(i \alpha_{1} h\right)\left(\alpha_{2} h\right)^{2}-135 x_{1} x_{2}\left(\alpha_{1} h\right)^{3}\left(\alpha_{2} h\right)-200 x_{1} x_{2}\left(\alpha_{1} h\right)^{2}\left(\alpha_{2} h\right)^{2} \\
& -12 x_{1} x_{2}\left(\alpha_{1} h\right)^{4}\left(i \alpha_{2} h\right)-90 x_{1} x_{2}\left(i \alpha_{1} h\right)^{3}\left(i \alpha_{2} h\right)^{2}+8 x_{1} x_{2}\left(\alpha_{1} h\right)^{4}\left(\alpha_{2} h\right)^{2} \\
& +150 x_{1}^{2} x_{2}\left(\alpha_{1} h\right)^{2}\left(i \alpha_{2} h\right)-135 x_{1}^{2} x_{2}\left(\alpha_{1} h\right)^{3}\left(\alpha_{2} h\right)-100 x_{1}^{2} x_{2}\left(\alpha_{1} h\right)^{2}\left(\alpha_{2} h\right)^{2} \\
& -18 x_{1}^{2} x_{2}\left(\alpha_{1} h\right)^{4}\left(i \alpha_{2} h\right)-90 x_{1}^{2} x_{2}\left(i \alpha_{1} h\right)^{3}\left(i \alpha_{2} h\right)^{2}+12 x_{1}^{2} x_{2}\left(\alpha_{1} h\right)^{4}\left(\alpha_{2} h\right)^{2} \\
& -45 x_{1}^{3} x_{2}\left(\alpha_{1} h\right)^{3}\left(\alpha_{2} h\right)-12 x_{1}^{3} x_{2}\left(\alpha_{1} h\right)^{4}\left(i \alpha_{2} h\right)-30 x_{1}^{3} x_{2}\left(i \alpha_{1} h\right)^{3}\left(i \alpha_{2} h\right)^{2} \\
& +8 x_{1}^{3} x_{2}\left(\alpha_{1} h\right)^{4}\left(\alpha_{2} h\right)^{2}-3 x_{1}^{4} x_{2}\left(\alpha_{1} h\right)^{4}\left(i \alpha_{2} h\right)+2 x_{1}^{4} x_{2}\left(\alpha_{1} h\right)^{4}\left(\alpha_{2} h\right)^{2} \\
& +60 x_{2}^{2}\left(i \alpha_{1} h\right)\left(\alpha_{2} h\right)^{2}-50 x_{2}^{2}\left(\alpha_{1} h\right)^{2}\left(\alpha_{2} h\right)^{2}-15 x_{2}^{2}\left(i \alpha_{1} h\right)^{3}\left(i \alpha_{2} h\right)^{2}+x_{2}^{2}\left(\alpha_{1} h\right)^{4}\left(\alpha_{2} h\right)^{2} \\
& +60 x_{1} x_{2}^{2}\left(i \alpha_{1} h\right)\left(\alpha_{2} h\right)^{2}-100 x_{1} x_{2}^{2}\left(\alpha_{1} h\right)^{2}\left(\alpha_{2} h\right)^{2}-45 x_{1} x_{2}^{2}\left(i \alpha_{1} h\right)^{3}\left(i \alpha_{2} h\right)^{2} \\
& +4 x_{1} x_{2}^{2}\left(\alpha_{1} h\right)^{4}\left(\alpha_{2} h\right)^{2}-50 x_{1}^{2} x_{2}^{2}\left(\alpha_{1} h\right)^{2}\left(\alpha_{2} h\right)^{2}-45 x_{1}^{2} x_{2}^{2}\left(i \alpha_{1} h\right)^{3}\left(i \alpha_{2} h\right)^{2} \\
& +6 x_{1}^{2} x_{2}^{2}\left(\alpha_{1} h\right)^{4}\left(\alpha_{2} h\right)^{2}-15 x_{1}^{3} x_{2}^{2}\left(i \alpha_{1} h\right)^{3}\left(i \alpha_{2} h\right)^{2}+4 x_{1}^{3} x_{2}^{2}\left(\alpha_{1} h\right)^{4}\left(\alpha_{2} h\right)^{2} \\
& +x_{1}^{4} x_{2}^{2}\left(\alpha_{1} h\right)^{4}\left(\alpha_{2} h\right)^{2} \text {, }
\end{aligned}
$$

where $r, \alpha_{1}, \alpha_{2}, x_{1}, x_{2}, y_{1}, y_{2}, z_{1}, z_{2}, w_{1}, w_{2}, u_{1}, u_{2}$ are defined by (88), (89), (93)-(95), (127) and (128).

\section{Appendix B}

Here, we present the center-corrected quadrature formulae of orders 4, 6 and 8 for the integral

$$
\int_{-a}^{a} \int_{-a}^{a} \phi(x, y) \cdot H_{0}\left(k \sqrt{x^{2}+y^{2}}\right) d x d y
$$

Lemma 6.6 (The $4^{\text {th }}$-Order Center-corrected Quadrature Formula). Suppose that $n \geq 1$ is an integer, and $a, h$ are two positive real numbers such that $h=a / n$. Suppose further that $\phi: \mathbb{R}^{2} \rightarrow \mathbb{C}$ is a function such that $\phi \in c^{2}(R \times R)$, and that $\phi$ is zero outside the square $[-a, a] \times[-a, a]$. Then, for any $k \in \mathbb{C}^{+}$,

$$
\int_{-a}^{a} \int_{-a}^{a} \phi(x, y) \cdot H_{0}\left(k \sqrt{x^{2}+y^{2}}\right) d x d y=U_{\tau^{h}}\left(\phi(x, y) \cdot H_{0}\left(k \sqrt{x^{2}+y^{2}}\right)\right)+O\left(h^{4}\right) .
$$

In (138),

$$
U_{\tau^{h}}\left(\phi(x, y) \cdot H_{0}\left(k \sqrt{x^{2}+y^{2}}\right)\right)=T^{\prime h}\left(\phi(x, y) \cdot H_{0}\left(k \sqrt{x^{2}+y^{2}}\right)\right)+\sum_{p, q \in S} \tau_{p q}^{h} \phi(p h, q h),
$$


where

$$
\begin{gathered}
S=\{p, q \in Z: p=0 \text { and } q=0\}, \\
T^{\prime h}\left(\phi(x, y) \cdot H_{0}\left(k \sqrt{x^{2}+y^{2}}\right)\right)=\sum_{(p, q) \neq(0,0)}\left(\phi(p h, q h) \cdot H_{0}\left(k \sqrt{(p h)^{2}+(q h)^{2}}\right)\right) \cdot h^{2},
\end{gathered}
$$

and

$$
\tau_{00}^{h}=D_{0} .
$$

Lemma 6.7 ( The $6^{\text {th }}$-Order Center-corrected Quadrature Formula). Suppose that $n \geq 1$ is an integer, and $a, h$ are two positive real numbers such that $h=a / n$. Suppose further that $\phi: \mathbb{R}^{2} \rightarrow \mathbb{C}$ is a function such that $\phi \in c^{4}(R \times R)$, and that $\phi$ is zero outside the square $[-a, a] \times[-a, a]$. Then, for any $k \in \mathbb{C}^{+}$,

$$
\int_{-a}^{a} \int_{-a}^{a} \phi(x, y) \cdot H_{0}\left(k \sqrt{x^{2}+y^{2}}\right) d x d y=U_{\tau^{h}}\left(\phi(x, y) \cdot H_{0}\left(k \sqrt{x^{2}+y^{2}}\right)\right)+O\left(h^{6}\right) .
$$

In (143),

$$
U_{\tau^{h}}\left(\phi(x, y) \cdot H_{0}\left(k \sqrt{x^{2}+y^{2}}\right)\right)=T^{\prime h}\left(\phi(x, y) \cdot H_{0}\left(k \sqrt{x^{2}+y^{2}}\right)\right)+\sum_{p, q \in S} \tau_{p q}^{h} \phi(p h, q h),
$$

where

$$
\begin{gathered}
S=\{p, q \in Z:|p+q| \leq 1 \text { and }|p-q| \leq 1\}, \\
T^{\prime h}\left(\phi(x, y) \cdot H_{0}\left(k \sqrt{x^{2}+y^{2}}\right)\right)=\sum_{(p, q) \neq(0,0)}\left(\phi(p h, q h) \cdot H_{0}\left(k \sqrt{(p h)^{2}+(q h)^{2}}\right)\right) \cdot h^{2},
\end{gathered}
$$

and

$$
\begin{gathered}
\tau_{00}^{h}=D_{0}-2 \frac{D_{1}}{h^{2}}, \\
\tau_{ \pm 10}^{h}=\tau_{0 \pm 1}^{h}=\frac{1}{2} \frac{D_{1}}{h^{2}} .
\end{gathered}
$$

Lemma 6.8 ( The $8^{\text {th }}$-Order Center-corrected Quadrature Formula). Suppose that $n \geq 1$ is an integer, and $a, h$ are two positive real numbers such that $h=a / n$. Suppose further that $\phi: \mathbb{R}^{2} \rightarrow \mathbb{C}$ is a function such that $\phi \in c^{6}(R \times R)$, and that $\phi$ is zero outside the square $[-a, a] \times[-a, a]$. Then, for any $k \in \mathbb{C}^{+}$,

$$
\int_{-a}^{a} \int_{-a}^{a} \phi(x, y) \cdot H_{0}\left(k \sqrt{x^{2}+y^{2}}\right) d x d y=U_{\tau^{h}}\left(\phi(x, y) \cdot H_{0}\left(k \sqrt{x^{2}+y^{2}}\right)\right)+O\left(h^{8}\right) .
$$

In (149),

$$
U_{\tau^{h}}\left(\phi(x, y) \cdot H_{0}\left(k \sqrt{x^{2}+y^{2}}\right)\right)=T^{\prime h}\left(\phi(x, y) \cdot H_{0}\left(k \sqrt{x^{2}+y^{2}}\right)\right)+\sum_{p, q \in S} \tau_{p q}^{h} \phi(p h, q h),
$$

where

$$
S=\{p, q \in Z:|p+q| \leq 2 \text { and }|p-q| \leq 2\}
$$




$$
T^{\prime h}\left(\phi(x, y) \cdot H_{0}\left(k \sqrt{x^{2}+y^{2}}\right)\right)=\sum_{(p, q) \neq(0,0)}\left(\phi(p h, q h) \cdot H_{0}\left(k \sqrt{(p h)^{2}+(q h)^{2}}\right)\right) \cdot h^{2},
$$

and

$$
\begin{gathered}
\tau_{00}^{h}=D_{0}-\frac{5}{2} \frac{D_{1}}{h^{2}}+\frac{1}{2} \frac{D_{2}}{h^{4}}+\frac{D_{3}}{h^{4}} \\
\tau_{ \pm 10}^{h}=\tau_{0 \pm 1}^{h}=\frac{2}{3} \frac{D_{1}}{h^{2}}-\frac{1}{6} \frac{D_{2}}{h^{4}}-\frac{1}{2} \frac{D_{3}}{h^{4}} \\
\tau_{ \pm 20}^{h}=\tau_{0 \pm 2}^{h}=-\frac{1}{24} \frac{D_{1}}{h^{2}}+\frac{1}{24} \frac{D_{2}}{h^{4}} \\
\tau_{ \pm 1 \pm 1}^{h}=\frac{1}{4} \frac{D_{3}}{h^{4}}
\end{gathered}
$$

\section{References}

[1] M. Abramowitz and I. Stegun, Handbook of Mathematical Functions, Applied Math. Series (National Bureau of Standards), Washington, DC, 1964.

[2] W. L. Briggs and V. E. Henson, The DFT: An Owners' Manual for the Discrete Fourier Transform, SIAM, Philadelphia, 1995.

[3] Y. Chen, A Fast Direct Algorithm for the Lippmann-Schwinger Integral Equation in Two Dimensions, Advances in Computational Mathematics. Vol. 16, pp. 175-190, 2002.

[4] D. L. Colton, R. Kress, Integral equation methods in scattering theory, Pure and Applied Mathematics, 1983.

[5] D. L. Colton, R. Kress, Inverse Acoustic and Electromagnetic Scattering Theory, Springer-Verlag, 1992.

[6] P. Ewald, Die Berechnung Optischer und Eletrostatischer Gitterpotentiale, Annals of Physics. Vol. 64, pp. 253-287, 1921.

[7] S. Kapur, V. Rokhlin, High-Order Corrected Trapezoidal Quadrature Rule for Singular Functions, Journal of Numerical Analysis, Vol. 34, No. 4, pp.1331-1356, 1997.

[8] J. Ma, Interpolation on Arbitrary Regions in the Complex Plane, Applied and Computational Harmonic Analysis, Vol. 4, Issue. 3, pp.264-292, 1997.

[9] P. M. Morse, H. Feshbach, Methods of Theoretical Physics, McGraw-Hill Book Company, USA, 1953.

[10] Y. Saad, Iterative Methods for Sparse Linear Systems, SIAM, USA, 2003.

[11] J. Stoer, R. Bulirsch, Introduction to Numerical Analysis, Springer-Verlag New York, Inc., USA, 1993.

[12] H. A. van der Vorst, BI-CGSTAB: a Fast and Smoothly Converging Variant of BI$C G$ for the Solution of Nonsymmetric Linear Systems, SIAM Journal on Scientific and Statistical Computing. Vol. 13, No. 2, pp.631-644, 1992. 